JOURNAL OF

FUNCTION SPACES AND APPLICATIONS

Volume 3, Number 2 (2005), 125-161
(C) 2005, Scientific Horizon http://www.jfsa.net

\title{
Two-scale convergence with respect to measures and homogenization of monotone operators
}

\author{
Dag Lukkassen and Peter Wall \\ (Communicated by Lars-Erik Persson)
}

2000 Mathematics Subject Classification. 35B27, 35B40.

Keywords and phrases. Homogenization, two-scale convergence, monotone operators, measure.

Abstract. In 1989 Nguetseng introduced two-scale convergence, which now is a frequently used tool in homogenization of partial differential operators. In this paper we discuss the notion of two-scale convergence with respect to measures. We make an exposition of the basic facts of this theory and develope it in various ways. In particular, we consider both variable $L^{p}$ spaces and variable Sobolev spaces. Moreover, we apply the results to a homogenization problem connected to a class of monotone operators.

\section{Introduction}

Let $\Omega$ be a bounded open subset of $\mathbb{R}^{N}$, where the Lebesgue measure of the boundary is zero, $Y=[0,1)^{N}$ the semi-open cube in $\mathbb{R}^{N}$ and $(\varepsilon)$ a sequence of positive numbers converging to 0 . In 1989 G. Nguetseng, see [7], proved that for each bounded sequence $\left(u_{\varepsilon}\right)$ in $L^{2}(\Omega)$ there exists a subsequence, still indexed by $\varepsilon$, and a $u \in L^{2}(\Omega \times Y)$ such that

$$
\int_{\Omega} u_{\varepsilon}(x) \phi\left(x, \frac{x}{\varepsilon}\right) d x \rightarrow \int_{\Omega} \int_{Y} u(x, y) \phi(x, y) d y d x,
$$


for every sufficiently smooth $\phi(x, y)$ which is $Y$-periodic in $y$. Nguetseng also proved that for a bounded sequence $\left(u_{\varepsilon}\right)$ in $W^{1,2}(\Omega)$ there exist functions $u \in L^{2}(\Omega \times Y)$ and $u_{1} \in L^{2}\left(\Omega ; W_{p e r}^{1,2}(Y)\right)$ such that, up to a subsequence,

$$
\begin{aligned}
u_{\varepsilon} & \rightarrow u \quad \text { weakly in } W^{1,2}(\Omega), \\
\int_{\Omega}\left(D u_{\varepsilon}(x), \Phi\left(x, \frac{x}{\varepsilon}\right)\right) d x & \rightarrow \int_{\Omega} \int_{Y}\left(D u(x)+D_{y} u_{1}(x, y), \Phi(x, y)\right) d y d x,
\end{aligned}
$$

for every sufficiently smooth $\Phi(x, y)$. The latter result made it possible for Nguetseng to make a new proof of the homogenization result corresponding to the linear elliptic problems

$$
\begin{cases}-\operatorname{div}\left(A\left(\frac{x}{\varepsilon}\right) D u_{\varepsilon}\right)=f & \text { on } \Omega, \\ u_{\varepsilon}=0 & \text { on } \partial \Omega,\end{cases}
$$

where $A$ is a $Y$-periodic matrix.

Later on G. Allaire, see [1], started to call the type of convergence defined by (1) two-scale convergence (or weak two-scale convergence). Allaire also developed the theory further by studying some general properties of twoscale convergence. Moreover he used two-scale convergence to analyze several homogenization problems, both linear and nonlinear. Two-scale convergence is now a well-known concept among people who work with homogenization. For a self-contained presentation of two-scale convergence we recommend the paper by Nguetseng et al. [6], which also include an overview of the main homogenization problems which have been studied by this technique.

In [13] V.V. Zhikov introduced the concept of weak (and strong) twoscale convergence with respect to a fixed periodic Borel measure $\mu$. More precisely, let $\mu$ be a $Y$-periodic positive Borel measure on $\mathbb{R}^{N}$ such that $\int_{Y} d \mu=1$. For any Borel set in $\mathbb{R}^{N}$ define

$$
\mu_{\varepsilon}(B)=\varepsilon^{N} \mu\left(\varepsilon^{-1} B\right), \quad \varepsilon^{-1} B=\{x / \varepsilon: x \in B\} .
$$

Then $u_{\varepsilon}$ in $L^{2}\left(\Omega, d \mu_{\varepsilon}\right)$ is said to weakly two-scale converge to $u \in L^{2}(\Omega \times Y$, $d x \times d \mu$ ) with respect to the measure $\mu$ if

$$
\int_{\Omega} u_{\varepsilon}(x) \phi\left(x, \frac{x}{\varepsilon}\right) d \mu_{\varepsilon} \rightarrow \int_{\Omega} \int_{Y} u(x, y) \phi(x, y) d x d \mu,
$$

for any sufficiently smooth $\phi(x, y)$ which is $Y$-periodic in $y$. We remark that in the case when $d \mu=d y$ is the Lebesgue measure on the cell of periodicity, the weak two-scale convergence by Nguetseng is obtained. In this setting Zhikov studied e.g. homogenization problems connected to degenerate elliptic operators, perforated domains and periodic graphs (for other works in this direction, see e.g. [2], [11], [12] and [14]). Recently 
Zhikov studied two-scale convergence with respect to measures in variable $L^{p}$-spaces for $p>1$, see [15].

In this paper we make a systematic exposition of the properties of weak and strong two-scale convergence with respect to measures both in variable $L^{p}$-spaces and variable Sobolev spaces (the measure is variable) for $p>1$. Finally, the developed two-scale technique is used to study a homogenization problem of the form:

$$
-\operatorname{div}\left(a\left(x / \varepsilon, D u_{\varepsilon}\right)\right)+\lambda\left|u_{\varepsilon}\right|^{p-2} u_{\varepsilon}=f_{\varepsilon}, \quad\left(u_{\varepsilon}, D u_{\varepsilon}\right) \in W_{0}^{1, p}\left(\Omega, d \mu_{\varepsilon}\right),
$$

where $a$ satisfies suitable continuity and monotonicity assumptions (the precise meaning this problem will be given later).

\section{Preliminaries and notation}

It will always be assumed that $p$ and $q$ are conjugate indexes, i.e. $1 / p+1 / q=1$ and that $1<p<\infty$. We note that $\mu_{\varepsilon}$ is a $\varepsilon$-periodic measure on $\mathbb{R}^{N}$ and that

$$
\mu_{\varepsilon}(\varepsilon B)=\varepsilon^{N} \int_{Y} d \mu=\varepsilon^{N} .
$$

Moreover, the measure $\mu_{\varepsilon}$ is weakly convergent to the Lebesgue measure (see Theorem 1 below), i.e.

$$
\int_{\mathbb{R}^{N}} \phi d \mu_{\varepsilon} \rightarrow \int_{\mathbb{R}^{N}} \phi d x, \quad \forall \phi \in C_{0}^{\infty}\left(\mathbb{R}^{N}\right) .
$$

We recall that a sequence $\left(u_{\varepsilon}\right)$ in $L^{p}(\Omega, d x)=L^{p}(\Omega)$ is weakly convergent to $u \in L^{p}(\Omega)$ if

$$
\int_{\Omega} u_{\varepsilon} v d x \rightarrow \int_{\Omega} u v d x, \quad \forall v \in L^{q}(\Omega) .
$$

We also recall the following well known facts concerning weak convergence in $L^{p}(\Omega)$ :

1. A weakly convergent sequence is bounded.

2. If a sequence $\left(u_{\varepsilon}\right)$ is bounded $L^{p}(\Omega)$ and

$$
\int_{\Omega} u_{\varepsilon} \phi d x \rightarrow \int_{\Omega} u \phi d x, \quad \forall \phi \in C_{0}^{\infty}(\Omega),
$$

then $u_{\varepsilon} \rightarrow u$ weakly in $L^{p}(\Omega)$.

3. A bounded sequence contains a weakly convergent sequence.

4. If $u_{\varepsilon} \rightarrow u$ weakly in $L^{p}(\Omega)$ and $g_{\varepsilon} \rightarrow g$ strongly in $L^{q}(\Omega)$, then

$$
\int_{\Omega} u_{\varepsilon} g_{\varepsilon} d x \rightarrow \int_{\Omega} u g d x
$$


5. Weak convergence of $\left(u_{\varepsilon}\right)$ to $u$ in $L^{p}(\Omega)$ together with

$$
\int_{\Omega}\left|u_{\varepsilon}\right|^{p} d x \rightarrow \int_{\Omega}|u|^{p} d x
$$

is equivalent to strong convergence of $\left(u_{\varepsilon}\right)$ to $u$ in $L^{p}(\Omega)$.

Let us now consider a sequence of functions $u_{\varepsilon} \in L^{p}\left(\Omega, d \mu_{\varepsilon}\right)$. By definition $\left(u_{\varepsilon}\right)$ is bounded in $L^{p}\left(\Omega, d \mu_{\varepsilon}\right)$ if $\int_{\Omega}\left|u_{\varepsilon}\right|^{p} d \mu_{\varepsilon} \leq c<\infty$. We say that a bounded sequence $\left(u_{\varepsilon}\right)$ in $L^{p}\left(\Omega, d \mu_{\varepsilon}\right)$ is weakly convergent to $u \in L^{p}(\Omega, d x)$, if

$$
\int_{\Omega} u_{\varepsilon} \phi d \mu_{\varepsilon} \rightarrow \int_{\Omega} u \phi d x \text { for any } \phi \in C_{0}^{\infty}(\Omega) .
$$

Moreover, a bounded sequence $\left(u_{\varepsilon}\right)$ in $L^{p}\left(\Omega, d \mu_{\varepsilon}\right)$ is defined to be strongly convergent to $u \in L^{p}(\Omega, d x)$, if

$$
\int_{\Omega} u_{\varepsilon} v_{\varepsilon} d \mu_{\varepsilon} \rightarrow \int_{\Omega} u v d x
$$

for every sequence $\left(v_{\varepsilon}\right)$ in $L^{q}\left(\Omega, d \mu_{\varepsilon}\right)$ which is weakly convergent to $v \in L^{q}(\Omega, d x)$. We have the following convergence properties in variable $L^{p}\left(\Omega, d \mu_{\varepsilon}\right)$ spaces:

1. If $\left(u_{\varepsilon}\right)$ is a bounded sequence in $L^{p}\left(\Omega, d \mu_{\varepsilon}\right)$, then $\left(u_{\varepsilon}\right)$ has a subsequence which is weakly convergent.

2. Weak convergence of $\left(u_{\varepsilon}\right)$ in $L^{p}\left(\Omega, d \mu_{\varepsilon}\right)$ to $u \in L^{p}(\Omega)$ and

$$
\int_{\Omega}\left|u_{\varepsilon}\right|^{p} d \mu_{\varepsilon} \rightarrow \int_{\Omega}|u|^{p} d x
$$

is equivalent to strong convergence of $\left(u_{\varepsilon}\right)$ in $L^{p}\left(\Omega, d \mu_{\varepsilon}\right)$ to $u \in L^{p}(\Omega)$.

3 . If the sequence $\left(u_{\varepsilon}\right)$ in $L^{p}\left(\Omega, d \mu_{\varepsilon}\right)$ converges strongly to $u$, where $u$ is a bounded continuous function on $\Omega$, then

$$
\int_{\Omega}\left|u_{\varepsilon}-u\right|^{p} d \mu_{\varepsilon} \rightarrow 0
$$

The proofs of these statements are closely related to the ones in next section where we consider weak two-scale convergence in variable $L^{p}$ spaces and are therefore left to the reader. We also encourage the reader to have these properties concerning weak convergence in variable $L^{p}\left(\Omega, d \mu_{\varepsilon}\right)$ spaces in mind during the reading of the section about two-scale convergence in variable $L^{p}\left(\Omega, d \mu_{\varepsilon}\right)$ and make relevant comparisons.

\section{Two-scale convergence in variable $L^{p}$ spaces}

Let us first state the following useful mean value property: 
Theorem 1 (Mean value property). Let $\phi(x, y)=\psi(x) \sigma(y)$, where $\psi$ is a bounded continuous function on $\Omega$ and $\sigma \in L_{\text {per }}^{1}(Y, d \mu)$. Then

$$
\int_{\Omega} \phi\left(x, \frac{x}{\varepsilon}\right) d \mu_{\varepsilon} \rightarrow \int_{\Omega} \int_{Y} \phi(x, y) d \mu(y) d x \quad \text { as } \varepsilon \rightarrow 0 .
$$

Proof. Assume that $\sigma \geq 0$. Let $Y_{k}=Y+k$, where $k$ is a vector in $\mathbb{R}^{N}$ with integer components. Then $\left\{\varepsilon Y_{k}\right\}$ is a partition of $\mathbb{R}^{N}$ and

$$
\int_{\Omega} \psi(x) \sigma\left(\frac{x}{\varepsilon}\right) d \mu_{\varepsilon}=\sum \int_{\varepsilon Y_{k}} \psi(x) \sigma\left(\frac{x}{\varepsilon}\right) d \mu_{\varepsilon}+\sum \int_{\varepsilon Y_{k} \cap \Omega} \psi(x) \sigma\left(\frac{x}{\varepsilon}\right) d \mu_{\varepsilon},
$$

where the first sum is taken over all $k$ such that $\varepsilon Y_{k}$ is inside $\Omega$ and the second sum is over all $k$ such that $\varepsilon Y_{k}$ and $\partial \Omega$ have common points. Let us first consider the first sum in (4). Since $\psi$ is continuous and bounded there exists points $x_{k} \in \varepsilon Y_{k}$ such that

$$
\int_{\varepsilon Y_{k}} \psi(x) \sigma\left(\frac{x}{\varepsilon}\right) d \mu_{\varepsilon}=\psi\left(x_{k}\right) \int_{\varepsilon Y_{k}} \sigma\left(\frac{x}{\varepsilon}\right) d \mu_{\varepsilon}=\psi\left(x_{k}\right) \varepsilon^{N} \int_{Y} \sigma(y) d \mu .
$$

This together with the fact that $\sum \varepsilon^{N} \psi\left(x_{k}\right) \rightarrow \int_{\Omega} \psi(x) d x$ as $\varepsilon \rightarrow 0$ implies that

$$
\lim _{\varepsilon \rightarrow 0} \sum \int_{\varepsilon Y_{k}} \psi(x) \sigma\left(\frac{x}{\varepsilon}\right) d \mu_{\varepsilon}=\int_{\Omega} \int_{Y} \psi(x) \sigma(y) d \mu(y) d x .
$$

Let us now consider the second sum in (4).

$$
\left|\sum \int_{\varepsilon Y_{k} \cap \Omega} \psi(x) \sigma\left(\frac{x}{\varepsilon}\right) d \mu_{\varepsilon}\right| \leq \max _{x \in \Omega}|\psi(x)| \int_{Y}|\sigma(y)| d \mu \varepsilon^{N} M(\varepsilon),
$$

where $M(\varepsilon)$ is the number of cubes $\varepsilon Y_{k}$ containing the boundary of $\Omega$. Moreover, $\varepsilon^{N} M(\varepsilon) \rightarrow 0$, since the boundary of $\Omega$ has Lebesgue measure zero. Thus

$$
\lim _{\varepsilon \rightarrow 0} \sum \int_{\varepsilon Y_{k} \cap \Omega} \psi(x) \sigma\left(\frac{x}{\varepsilon}\right) d \mu_{\varepsilon}=0 .
$$

The desired relation (3) follows by taking (4)-(6) into account. The proof for $\sigma$ of arbitrary sign follows by dividing $\sigma$ into positive and negative parts and repeating the arguments above.

Let $D$ be the set of functions defined as

$$
D=\left\{\phi: \phi(x, y)=\sum_{\text {finite }} \psi_{i}(x) \sigma_{i}(y), \psi_{i} \in C_{0}^{\infty}(\Omega), \sigma_{i} \in C_{\text {per }}^{\infty}(Y)\right\} .
$$

Then Theorem 1 implies that

$$
\int_{\Omega} \phi\left(x, \frac{x}{\varepsilon}\right) d \mu_{\varepsilon} \rightarrow \int_{\Omega} \int_{Y} \phi(x, y) d \mu(y) d x \text { as } \varepsilon \rightarrow 0,
$$


for any $\phi \in D$. It is important to obtain a wide class of functions which satisfy the mean value property (3). One such important class is defined below.

Definition 1. Let $\mathcal{B}$ be the class of functions $\phi: \Omega \times \mathbb{R}^{N} \rightarrow \mathbb{R}$ which satisfies:

(a) The function $x \rightarrow \phi(x, y)$ is continuous for $\mu$-almost every $y$.

(b) The function $y \rightarrow \phi(x, y)$ is $\mu$-measurable and $Y$-periodic for every $x \in \Omega$.

(c) The function $y \rightarrow \sup _{x \in \Omega}|\phi(x, y)|$ is in $L_{\mathrm{per}}^{1}(Y, d \mu)$.

Theorem 2. Let $\phi \in \mathcal{B}$. Then

$$
\int_{\Omega} \phi\left(x, \frac{x}{\varepsilon}\right) d \mu_{\varepsilon} \rightarrow \int_{\Omega} \int_{Y} \phi(x, y) d \mu(y) d x \quad \text { as } \varepsilon \rightarrow 0 .
$$

Proof. From (a) and (b) in the definition of $\mathcal{B}$ we have that $\phi$ is of Carathedory type which assure the measurability of $\phi(x, x / \varepsilon)$. Let $A=[0,1)^{N}, A_{k}=A+k$, where $k$ is a vector in $\mathbb{R}^{N}$ with integer components. Then $\left\{1 / n A_{k}\right\}, n=1,2,3, \ldots$, is a partition of $\mathbb{R}^{N}$. We denote the characteristic function of $1 / n A_{k}$ by $\chi_{k}$ and $x_{k}$ is an arbitrary point in $1 / n A_{k}$. First we prove (8) for step functions $\phi_{n}: \Omega \times \mathbb{R}^{N} \rightarrow \mathbb{R}$ of the form

$$
\phi_{n}(x, y)=\sum \phi\left(x_{k}, y\right) \chi_{k}(x)
$$

where the sum is taken over $k$ such that $1 / n A_{k} \subset \Omega$. We note that the function $y \rightarrow \phi\left(x_{k}, y\right)$ is in $L_{\mathrm{per}}^{1}(Y, d \mu)$. By Theorem 1 it follows that

$$
\begin{aligned}
\lim _{\varepsilon \rightarrow 0} \int_{\Omega} \phi_{n}\left(x, \frac{x}{\varepsilon}\right) d \mu_{\varepsilon} & =\sum_{\varepsilon \rightarrow 0} \int_{1 / n A_{k}} \phi\left(x_{k}, \frac{x}{\varepsilon}\right) d \mu_{\varepsilon} \\
& =\int_{\Omega} \int_{Y} \phi_{n}(x, y) d \mu(y) d x .
\end{aligned}
$$

By using (9) we obtain that

$$
\begin{aligned}
\lim _{\varepsilon \rightarrow 0}\left|\int_{\Omega} \phi\left(x, \frac{x}{\varepsilon}\right) d \mu_{\varepsilon}-\int_{\Omega} \int_{Y} \phi(x, y) d \mu(y) d x\right| \\
\leq \lim _{\varepsilon \rightarrow 0}\left|\int_{\Omega} \phi\left(x, \frac{x}{\varepsilon}\right)-\phi_{n}\left(x, \frac{x}{\varepsilon}\right) d \mu_{\varepsilon}\right| \\
\quad+\lim _{\varepsilon \rightarrow 0}\left|\int_{\Omega} \phi_{n}\left(x, \frac{x}{\varepsilon}\right) d \mu_{\varepsilon}-\int_{\Omega} \int_{Y} \phi_{n}(x, y) d \mu(y) d x\right| \\
\quad+\left|\int_{\Omega} \int_{Y} \phi_{n}(x, y) d \mu(y) d x-\int_{\Omega} \int_{Y} \phi(x, y) d \mu(y) d x\right|
\end{aligned}
$$




$$
\begin{aligned}
= & \lim _{\varepsilon \rightarrow 0} \int_{\Omega}\left|\phi\left(x, \frac{x}{\varepsilon}\right)-\phi_{n}\left(x, \frac{x}{\varepsilon}\right)\right| d \mu_{\varepsilon} \\
& +\int_{\Omega} \int_{Y}\left|\phi_{n}(x, y)-\phi(x, y)\right| d \mu(y) d x \\
\leq & 2|\Omega| \int_{Y} \sup _{x \in \Omega}\left|\phi(x, y)-\phi_{n}(x, y)\right| d \mu(y),
\end{aligned}
$$

for every $n \in \mathbb{N}$. The proof is complete if we show that the right hand side in (10) tends to 0 as $n \rightarrow \infty$. Since $\phi(x, y)$ is continuous in $x$ for $\mu$-almost every $y$ we have that

$$
g_{n}(y)=\sup _{x \in \Omega}\left|\phi_{n}(x, y)-\phi(x, y)\right| \rightarrow 0 \text { for a.e. } y \in Y .
$$

Moreover,

$$
g_{n}(y) \leq 2 \sup _{x \in \Omega}|\phi(x, y)| \in L_{\text {per }}^{1}(Y, d \mu) .
$$

The Lebesgue dominated convergence theorem implies that

$$
\int_{Y} \sup _{x \in \Omega}\left|\phi(x, y)-\phi_{n}(x, y)\right| d \mu(y) \rightarrow 0 \quad \text { as } n \rightarrow \infty .
$$

Definition 2. Let $\left(u_{\varepsilon}\right)$ be a bounded sequence in $L^{p}\left(\Omega, d \mu_{\varepsilon}\right)$ and $u \in$ $L^{p}(\Omega \times Y, d x \times d \mu)=L^{p}(\Omega \times Y)$. Then we say that $\left(u_{\varepsilon}\right)$ two-scale converges weakly to $u$ (we write $u_{\varepsilon} \stackrel{2}{\rightarrow} u$ ) if

$$
\int_{\Omega} u_{\varepsilon}(x) \phi\left(x, \frac{x}{\varepsilon}\right) d \mu_{\varepsilon} \rightarrow \int_{\Omega} \int_{Y} u(x, y) \phi(x, y) d \mu(y) d x \text { as } \varepsilon \rightarrow 0,
$$

for every test function $\phi$ of the form $\phi(x, y)=\psi(x) \sigma(y)$, where $\psi \in C_{0}^{\infty}(\Omega)$ and $\sigma \in C_{\text {per }}^{\infty}(Y)$.

We note that we have an equivalent definition of two-scale convergence if we replace the set of test functions by $D(D$ defined as in $(7))$. As a direct consequence of the definition of weak two-scale convergence we have that if $\left(u_{\varepsilon}\right)$ is a bounded sequence in $L^{p}\left(\Omega, d \mu_{\varepsilon}\right)$ which two-scale converges weakly to $u \in L^{p}(\Omega \times Y, d x \times d \mu)$, then $\left(u_{\varepsilon}\right)$ converges weakly to $v \in L^{p}(\Omega, d x)$, where $v(x)=\int_{Y} u(x, y) d \mu(y)$. This fact follows by by choosing test functions independent of $y$ in (11).

Theorem 3. If $\left(u_{\varepsilon}\right)$ is a bounded sequence in $L^{p}\left(\Omega, d \mu_{\varepsilon}\right)$, then there exists a subsequence which two-scale converges weakly.

Proof. Let $\phi \in D(D$ defined as in (7)). By Hölder's inequality and the fact that $\left(u_{\varepsilon}\right)$ is a bounded sequence in $L^{p}\left(\Omega, d \mu_{\varepsilon}\right)$ we get that

$$
\left|\int_{\Omega} u_{\varepsilon}(x) \phi\left(x, \frac{x}{\varepsilon}\right) d \mu_{\varepsilon}\right| \leq c\left\|\phi\left(x, \frac{x}{\varepsilon}\right)\right\|_{L^{q}\left(\Omega, d \mu_{\varepsilon}\right)} \leq c \mu_{\varepsilon}(\Omega)^{1 / q}\|\phi\|_{\max _{\Omega \times Y}} .
$$


This means that $u_{\varepsilon}$ can be identified with an element $U_{\varepsilon}$ in the dual space $D^{*}$ of $D$ through the formula

$$
\left\langle U_{\varepsilon}, \phi\right\rangle=\int_{\Omega} u_{\varepsilon}(x) \phi\left(x, \frac{x}{\varepsilon}\right) d x .
$$

From (12) it follows that

$$
\left\|U_{\varepsilon}\right\|_{D^{*}}=\sup _{\|\phi\|_{\max _{\Omega \times Y}=1}}\left|\left\langle U_{\varepsilon}, \phi\right\rangle\right| \leq c \mu_{\varepsilon}(\Omega)^{1 / q} .
$$

Applying lim sup on both sides in this inequality and taking Theorem 1 into account in the right hand side gives that limsup $\left\|U_{\varepsilon}\right\|_{D^{*}} \leq c|\Omega|^{1 / q}$. Now we use the well-known result that each bounded sequence in the dual space of a separable normed space contains a subsequence (still denoted by $\varepsilon$ ) which converges weak-*. In our case this means that there exists a $U \in D^{*}$ such that

$$
\int_{\Omega} u_{\varepsilon}(x) \phi\left(x, \frac{x}{\varepsilon}\right) d x=\left\langle U_{\varepsilon}, \phi\right\rangle \rightarrow\langle U, \phi\rangle,
$$

for every $\phi \in D$. The proof will be complete if we can show that there exists an element $u \in L^{p}(\Omega \times Y, d x \times d \mu)$ such that

$$
\langle U, \phi\rangle=\int_{\Omega} \int_{Y} u(x, y) \phi(x, y) d \mu(y) d x .
$$

By (12) we have that

$$
\left|\left\langle U_{\varepsilon}, \phi\right\rangle\right| \leq c\left\|\phi\left(x, \frac{x}{\varepsilon}\right)\right\|_{L^{q}\left(\Omega, d \mu_{\varepsilon}\right)} .
$$

Moreover, by taking (13) and Theorem 2 into account and passing to the limit we get

$$
|\langle U, \phi\rangle| \leq c\|\phi\|_{L^{q}(\Omega \times Y)},
$$

for each $\phi \in D$. Since $D$ is dense in $L^{q}(\Omega \times Y)$ we can for each $\left.\phi \in L^{q}(\Omega \times Y, d x \times d \mu)\right)$ find a sequence $\left(\phi_{h}\right)$ in $D$ such that $\phi_{h} \rightarrow \phi$ in $L^{q}(\Omega \times Y, d x \times d \mu)$. This means that we can define an extension $\widetilde{U}$ of $U$ to $L^{q}(\Omega \times Y, d x \times d \mu)$ as

$$
\langle\widetilde{U}, \phi\rangle=\lim _{h \rightarrow 0}\left\langle U, \phi_{h}\right\rangle .
$$

Riesz representation theorem guarantees the existence of an $u \in L^{p}(\Omega \times Y$, $d x \times d \mu$ ) such that

$$
\langle\widetilde{U}, \phi\rangle=\int_{\Omega} \int_{Y} u(x, y) \phi(x, y) d y d x,
$$

for each $\phi$ in $L^{q}(\Omega \times Y)$. Especially, for $\phi$ in $D$ we have that

$$
\langle U, \phi\rangle=\langle\widetilde{U}, \phi\rangle=\int_{\Omega} \int_{Y} u(x, y) \phi(x, y) d y d x
$$

and the proof is complete. 
Theorem 4. Let $\left(u_{\varepsilon}\right)$ be a bounded sequence in $L^{p}\left(\Omega, d \mu_{\varepsilon}\right)$ which twoscale converges weakly to $u \in L^{p}(\Omega \times Y, d x \times d \mu)=L^{p}(\Omega \times Y)$. Then

$$
\liminf _{\varepsilon \rightarrow 0}\left\|u_{\varepsilon}\right\|_{L^{p}\left(\Omega, d \mu_{\varepsilon}\right)} \geq\|u\|_{L^{p}(\Omega \times Y)} \geq\|v\|_{L^{p}(\Omega, d x)},
$$

where $v(x)=\int_{Y} u(x, y) d \mu(y)$.

Proof. Let $\left(\phi_{m}\right)$ be a sequence in $D(D$ defined as in $(7))$ such that $\phi_{m}$ converges to $|u|^{p-2} u$ strongly in $L^{q}(\Omega \times Y)$. The Young inequality for real numbers $a$ and $b$ states that $a b \leq|a|^{p} / p+|b|^{q} / q$ and it implies that

$$
\int_{\Omega}\left|u_{\varepsilon}\right|^{p} d \mu_{\varepsilon} \geq p \int_{\Omega} u_{\varepsilon}(x) \phi_{m}\left(x, \frac{x}{\varepsilon}\right) d \mu_{\varepsilon}-(p-1) \int_{\Omega}\left|\phi_{m}\left(x, \frac{x}{\varepsilon}\right)\right|^{q} d \mu_{\varepsilon} .
$$

By passing to the limit in $\varepsilon$ we get

$$
\begin{aligned}
\liminf _{\varepsilon \rightarrow 0}\left\|u_{\varepsilon}\right\|_{L^{p}\left(\Omega, d \mu_{\varepsilon}\right)}^{p} \geq & p \int_{\Omega} \int_{Y} u(x, y) \phi_{m}(x, y) d \mu(y) d x \\
& -(p-1) \int_{\Omega} \int_{Y}\left|\phi_{m}(x, y)\right|^{q} d \mu(y) d x .
\end{aligned}
$$

Moreover, by also passing to the limit in $m$ we obtain

$$
\begin{aligned}
\liminf _{\varepsilon \rightarrow 0}\left\|u_{\varepsilon}\right\|_{L^{p}\left(\Omega, d \mu_{\varepsilon}\right)}^{p} & \geq p \int_{\Omega} \int_{Y}|u|^{p} d \mu d x-(p-1) \int_{\Omega} \int_{Y}|u|^{p} d \mu d x \\
& =\|u\|_{L^{p}(\Omega \times Y)}^{p} .
\end{aligned}
$$

Finally, by Jensen's inequality we have that

$$
\begin{aligned}
\|v\|_{L^{p}(\Omega, d x)}^{p} & =\int_{\Omega}\left|\int_{Y} u(x, y) d \mu(y)\right|^{p} d x \\
& \leq \int_{\Omega} \int_{Y}|u(x, y)|^{p} d \mu(y) d x \\
& =\|u\|_{L^{p}(\Omega \times Y)}^{p}
\end{aligned}
$$

and the proof is complete.

Definition 3. Let $\left(u_{\varepsilon}\right)$ be a bounded sequence in $L^{p}\left(\Omega, d \mu_{\varepsilon}\right)$ and $u \in$ $L^{p}(\Omega \times Y, d x \times d \mu)$. Then we say that $\left(u_{\varepsilon}\right)$ two-scale converges strongly to $u$ (we write $u_{\varepsilon} \stackrel{2}{\rightarrow} u$ ) if for any bounded sequence $\left(v_{\varepsilon}\right)$ in $L^{q}\left(\Omega, d \mu_{\varepsilon}\right)$ which two-scale converges weakly to $v \in L^{q}(\Omega \times Y)$, we have that

$$
\int_{\Omega} u_{\varepsilon}(x) v_{\varepsilon}(x) d \mu_{\varepsilon} \rightarrow \int_{\Omega} \int_{Y} u(x, y) v(x, y) d \mu(y) d x .
$$

Theorem 5. Weak two-scale convergence of the sequence $\left(u_{\varepsilon}\right)$ in $L^{p}\left(\Omega, d \mu_{\varepsilon}\right)$ to $u \in L^{p}(\Omega \times Y, d x \times d \mu)=L^{p}(\Omega \times Y)$ together with

$$
\lim _{\varepsilon \rightarrow 0} \int_{\Omega}\left|u_{\varepsilon}\right|^{p} d \mu_{\varepsilon}=\int_{\Omega} \int_{Y}|u|^{p} d \mu d x
$$


is equivalent to strong two-scale convergence of $\left(u_{\varepsilon}\right)$ to $u$, i.e. to that

$$
\int_{\Omega} u_{\varepsilon}(x) v_{\varepsilon}(x) d \mu_{\varepsilon} \rightarrow \int_{\Omega} \int_{Y} u(x, y) v(x, y) d \mu(y) d x
$$

for any sequence $\left(v_{\varepsilon}\right)$ in $L^{q}\left(\Omega, d \mu_{\varepsilon}\right)$ which two-scale converges weakly to $v \in L^{q}(\Omega \times Y)$.

Proof. (i) We start by proving that weak two-scale convergence together with (14) imply strong two-scale convergence. Let $\left(\phi_{m}\right)$ be a sequence in $D$ $\left(D\right.$ defined as in (7)) such that $\phi_{m}$ converges to $u$ strongly in $L^{p}(\Omega \times Y)$. Then, since $\left(v_{\varepsilon}\right)$ two-scale converges weakly to $v \in L^{q}(\Omega \times Y)$,

$$
\lim _{m \rightarrow \infty} \lim _{\varepsilon \rightarrow 0} \int_{\Omega} \phi_{m}\left(x, \frac{x}{\varepsilon}\right) v_{\varepsilon}(x) d \mu_{\varepsilon}=\int_{\Omega} \int_{Y} u(x, y) v(x, y) d \mu(y) d x .
$$

We also have that

$$
\begin{aligned}
& \left|\int_{\Omega} u_{\varepsilon}(x) v_{\varepsilon}(x) d \mu_{\varepsilon}-\int_{\Omega} \int_{Y} u(x, y) v(x, y) d \mu(y) d x\right| \\
& \leq\left|\int_{\Omega}\left[u_{\varepsilon}(x)-\phi_{m}\left(x, \frac{x}{\varepsilon}\right)\right] v_{\varepsilon}(x) d \mu_{\varepsilon}\right| \\
& \quad+\left|\int_{\Omega} \phi_{m}\left(x, \frac{x}{\varepsilon}\right) v_{\varepsilon}(x) d \mu_{\varepsilon}-\int_{\Omega} \int_{Y} u(x, y) v(x, y) d \mu(y) d x\right| .
\end{aligned}
$$

From (16) and (17) we obtain that

$$
\begin{aligned}
& \limsup _{\varepsilon \rightarrow 0}\left|\int_{\Omega} u_{\varepsilon}(x) v_{\varepsilon}(x) d \mu_{\varepsilon}-\int_{\Omega} \int_{Y} u(x, y) v(x, y) d \mu(y) d x\right| \\
& \leq \limsup _{m \rightarrow \infty} \limsup _{\varepsilon \rightarrow 0}\left|\int_{\Omega}\left[u_{\varepsilon}(x)-\phi_{m}\left(x, \frac{x}{\varepsilon}\right)\right] v_{\varepsilon}(x) d \mu_{\varepsilon}\right| .
\end{aligned}
$$

It is clear that (15) follows from (18) if we prove

$$
\limsup _{m \rightarrow \infty} \limsup _{\varepsilon \rightarrow 0}\left|\int_{\Omega}\left[u_{\varepsilon}(x)-\phi_{m}\left(x, \frac{x}{\varepsilon}\right)\right] v_{\varepsilon}(x) d \mu_{\varepsilon}\right|=0 .
$$

The Hölder inequality and the facts (by definition) that each weakly two scale convergent sequence is bounded imply

$$
\begin{aligned}
\left|\int_{\Omega}\left[u_{\varepsilon}(x)-\phi_{m}\left(x, \frac{x}{\varepsilon}\right)\right] v_{\varepsilon}(x) d \mu_{\varepsilon}\right| \leq & \left(\int_{\Omega}\left|u_{\varepsilon}(x)-\phi_{m}\left(x, \frac{x}{\varepsilon}\right)\right|^{p} d \mu_{\varepsilon}\right)^{1 / p} \\
& \times\left(\int_{\Omega}\left|v_{\varepsilon}(x)\right|^{q} d \mu_{\varepsilon}\right)^{1 / q} \\
& \leq c\left\|u_{\varepsilon}(x)-\phi_{m}\left(x, \frac{x}{\varepsilon}\right)\right\|_{L^{p}\left(\Omega, d \mu_{\varepsilon}\right)} .
\end{aligned}
$$


The Clarkson inequalities give, respectively, for $p \geq 2$ and for $p \leq 2$

$$
\begin{gathered}
\left\|u_{\varepsilon}-\phi_{m}\left(x, \frac{x}{\varepsilon}\right)\right\|^{p} \leq 2^{p}\left[\frac{1}{2}\left\|u_{\varepsilon}\right\|^{p}+\frac{1}{2}\left\|\phi_{m}\left(x, \frac{x}{\varepsilon}\right)\right\|^{p}-\left\|\frac{u_{\varepsilon}+\phi_{m}\left(x, \frac{x}{\varepsilon}\right)}{2}\right\|^{p}\right], \\
\left\|u_{\varepsilon}-\phi_{m}\left(x, \frac{x}{\varepsilon}\right)\right\|^{q} \leq 2^{p-1}\left[\left(\frac{1}{2}\left\|u_{\varepsilon}\right\|^{p}+\frac{1}{2}\left\|\phi_{m}\left(x, \frac{x}{\varepsilon}\right)\right\|^{p}\right)^{\frac{1}{p-1}}\right. \\
\left.-\left\|\frac{u_{\varepsilon}+\phi_{m}\left(x, \frac{x}{\varepsilon}\right)}{2}\right\|^{q}\right]
\end{gathered}
$$

where all norms are the usual norm in $L^{p}\left(\Omega, d \mu_{\varepsilon}\right)$. By (14) and an application of Theorem 1 (the mean value property) to the sequence $u_{\varepsilon}(x)+\phi_{m}(x, x / \varepsilon)$ (which two-scale converges weakly to $u+\phi_{m} \in L^{p}(\Omega \times Y)$ ) it follows for $p \geq 2$ that

$$
\begin{aligned}
& \text { (21) } \limsup _{\varepsilon \rightarrow 0}\left\|u_{\varepsilon}-\phi_{m}\left(x, \frac{x}{\varepsilon}\right)\right\|_{L^{p}\left(\Omega, d \mu_{\varepsilon}\right)}^{p} \\
& \quad \leq 2^{p}\left[\frac{1}{2}\|u\|_{L^{p}(\Omega \times Y)}^{p}+\frac{1}{2}\left\|\phi_{m}\right\|_{L^{p}(\Omega \times Y)}^{p}-\left\|\frac{u+\phi_{m}}{2}\right\|_{L^{p}(\Omega \times Y)}^{p}\right]
\end{aligned}
$$

and for $1<p \leq 2$ that

$$
\begin{aligned}
& \quad \limsup _{\varepsilon \rightarrow 0}\left\|u_{\varepsilon}-\phi_{m}\left(x, \frac{x}{\varepsilon}\right)\right\|_{L^{p}\left(\Omega, d \mu_{\varepsilon}\right)}^{q} \\
& \leq 2^{p-1}\left[\left(\frac{1}{2}\|u\|_{L^{p}(\Omega \times Y)}^{p}+\frac{1}{2}\left\|\phi_{m}\right\|_{L^{p}(\Omega \times Y)}^{p}\right)^{\frac{1}{p-1}}-\left\|\frac{u+\phi_{m}}{2}\right\|_{L^{p}(\Omega \times Y)}^{q}\right] .
\end{aligned}
$$

In view of the choice of $\phi_{m}$ we have that $\left(u+\phi_{m}\right) / 2$ converges to $u$ in $L^{p}(\Omega \times Y)$. By applying limsup on both sides in (21) and (22) we find that

$$
\limsup _{m \rightarrow \infty} \limsup _{\varepsilon \rightarrow 0}\left\|u_{\varepsilon}-\phi_{m}\left(x, \frac{x}{\varepsilon}\right)\right\|_{L^{p}\left(\Omega, d \mu_{\varepsilon}\right)}=0 .
$$

Now (19) follows from (20) and (23).

(ii) It remains to prove that strong two-scale convergence implies weak twoscale convergence and relation (14). Assume that $\left(u_{\varepsilon}\right)$ two-scale converges strongly to $u \in L^{p}(\Omega \times Y)$. By choosing $v_{\varepsilon}(x)=\psi(x) \sigma(x / \varepsilon)$, where $\psi \in C_{0}^{\infty}(\Omega)$ and $\sigma \in C_{\text {per }}^{\infty}(Y)$ in (15) we see that strong two-scale converge implies weak two-scale convergence. Now we will show that strong twoscale convergence implies (14). In fact, let $v_{\varepsilon}=\left|u_{\varepsilon}\right|^{p-2} u_{\varepsilon}$. By the definition of strong (and weak) two-scale convergence $\left(u_{\varepsilon}\right)$ is bounded in $L^{p}\left(\Omega, d \mu_{\varepsilon}\right)$ and hence $\left(v_{\varepsilon}\right)$ is bounded in $L^{q}\left(\Omega, d \mu_{\varepsilon}\right)$. By Theorem 3 there exists a subsequence (still denoted by $\varepsilon$ ) which two-scale converges weakly to $v \in L^{q}(\Omega \times Y)$. According to the definition of strong two-scale convergence 
we have

$$
\int_{\Omega}\left|u_{\varepsilon}\right|^{p} d \mu_{\varepsilon}=\int_{\Omega} u_{\varepsilon} v_{\varepsilon} d \mu_{\varepsilon} \rightarrow \int_{\Omega} \int_{Y} u(x, y) v(x, y) d \mu(y) d x .
$$

From this we see that we are done if we show that $v=|u|^{p-2} u$. In fact, the function $f(t)=|t|^{p-2} t$ is monotone which means that $\left[f\left(t_{1}\right)-f\left(t_{2}\right)\right]\left(t_{1}-\right.$ $\left.t_{2}\right) \geq 0$ for every $t_{1}, t_{2} \in \mathbb{R}^{N}$. Let $\phi(x, y)=\sum_{\text {finite }} \psi_{i}(x) \sigma_{i}(y)$, where $\psi_{i} \in C_{0}^{\infty}(\Omega), \sigma_{i} \in C_{\mathrm{per}}^{\infty}(Y)$. Then

$$
\int_{\Omega}\left[f(\phi(x, x / \varepsilon))-f\left(u_{\varepsilon}(x)\right)\right]\left(\phi(x, x / \varepsilon)-u_{\varepsilon}(x)\right) d \mu_{\varepsilon} \geq 0 .
$$

In the limit we get

$$
\int_{\Omega} \int_{Y}[f(\phi(x, y))-v(x, y)](\phi(x, y)-u(x, y)) d \mu d x \geq 0 .
$$

By density and continuity this inequality holds for any $\phi \in L^{p}(\Omega \times Y)$. Put $\phi=u+t w, w \in L^{p}(\Omega \times Y)$. For $t>0$ we get that

$$
\int_{\Omega} \int_{Y}\left[\mid u(x, y)+t w\left(x,\left.y\right|^{p-2}(u(x, y)+t w(x, y))-v(x, y)\right] w(x, y) d \mu d x \geq 0 .\right.
$$

For $t<0$ we obtain that

$$
\int_{\Omega} \int_{Y}\left[\mid u(x, y)+t w\left(x,\left.y\right|^{p-2}(u(x, y)+t w(x, y))-v(x, y)\right] w(x, y) d \mu d x \leq 0 .\right.
$$

By taking (24) and (25) into account and leting $t$ tend to 0 we find that

$$
\int_{\Omega} \int_{Y}\left[|u(x, y)|^{p-2} u(x, y)-v(x, y)\right] w(x, y) d \mu d x=0 \text { forall } w \in L^{p}(\Omega \times Y),
$$
which implies that $v=|u|^{p-2} u$. The proof is complete.

Let $\mathcal{A}_{q}$ be the set of functions $\phi$ in $\mathcal{B}(\mathcal{B}$ defined as in Definition 1) such that the function $y \rightarrow \sup _{x \in \Omega}|\phi(x, y)|$ is in $L_{\text {per }}^{q}(Y, d \mu)$. We note that $\mathcal{A}_{q} \subset L^{q}(\Omega \times Y)$ and that if $\phi \in \mathcal{A}_{q}$, then $\phi(x, y) \psi(x) \sigma(y) \in \mathcal{A}_{q} \subset \mathcal{B}$ for any $\psi \in C_{0}^{\infty}(\Omega)$ and any $\sigma \in C_{\text {per }}^{\infty}(Y)$. Theorem 2 implies the convergence

$$
\int_{\Omega} \phi\left(x, \frac{x}{\varepsilon}\right) \psi(x) \sigma\left(\frac{x}{\varepsilon}\right) d \mu_{\varepsilon} \rightarrow \int_{\Omega} \int_{Y} \phi(x, y) \psi(x) \sigma(y) d \mu(y) d x,
$$

i.e. $\phi(x, x / \varepsilon)$ two-scale converges weakly to $\phi(x, y)$ if $\phi \in \mathcal{A}_{q}$. If $\phi \in \mathcal{A}_{q}$, then $|\phi| \in \mathcal{A}_{q}$, and thus $|\phi|^{q} \in \mathcal{B}$. As a consequence of Theorem 2 we obtain that

$$
\lim _{\varepsilon \rightarrow 0} \int_{\Omega}\left|\phi\left(x, \frac{x}{\varepsilon}\right)\right|^{q} d \mu_{\varepsilon}=\int_{\Omega} \int_{Y}|\phi(x, y)|^{q} d \mu d x
$$


Moreover, we note that Theorem 5 together with (26) and (27) implies that if $\phi \in \mathcal{A}_{q}$, then $\phi(x, x / \varepsilon)$ two-scale converges strongly to $\phi(x, y) \in L^{q}(\Omega \times Y)$. Hence we have the following corollary of Theorem 5:

Corollary 6. If a sequence $\left(u_{\varepsilon}\right)$ in $L^{p}\left(\Omega, d \mu_{\varepsilon}\right)$ two-scale converges weakly to $u \in L^{p}(\Omega \times Y)$, then

$$
\int_{\Omega} u_{\varepsilon}(x) \phi\left(x, \frac{x}{\varepsilon}\right) d \mu_{\varepsilon} \rightarrow \int_{\Omega} \int_{Y} u(x, y) \phi(x, y) d \mu(y) d x
$$

for any $\phi \in \mathcal{A}_{q}$.

An other consequence of Theorem 5 is the following corollary:

Corollary 7. Let $\left(u_{\varepsilon}\right)$ be a bounded sequence in $L^{p}\left(\Omega, d \mu_{\varepsilon}\right)$ which twoscale converges weakly to $u \in L^{p}(\Omega \times Y, d x \times d \mu)=L^{p}(\Omega \times Y)$ and also satisfies that

$$
\lim _{\varepsilon \rightarrow 0} \int_{\Omega}\left|u_{\varepsilon}\right|^{p} d \mu_{\varepsilon}=\int_{\Omega} \int_{Y}|u|^{p} d \mu d x .
$$

If the limit $u$ belong to $\mathcal{A}_{p}$, then

$$
\int_{\Omega}\left|u_{\varepsilon}(x)-u(x, x / \varepsilon)\right|^{p} d \mu_{\varepsilon} \rightarrow 0 .
$$

Proof. By Theorem $5 u_{\varepsilon}$ two-scale converges strongly to $u \in L^{p}(\Omega \times Y)$. Since $u \in \mathcal{A}_{p}$ we also have that $u(x, x / \varepsilon)$ two-scale converges strongly to $u \in L^{p}(\Omega \times Y)$. Hence $u_{\varepsilon}(x)-u(x, x / \varepsilon)$ two scale converges strongly to 0 and (28) follows by taking Theorem 5 into account.

The lower semicontinuity property for weak two-scale convergence described in Theorem 4 may be generalized to the case of convex periodic integrands.

Theorem 8. Let $\left(v_{\varepsilon}\right)$ be a sequence in $L^{p}\left(\Omega, d \mu_{\varepsilon}\right)^{N}$ which two-scale converges weakly (by components) to $v \in L^{p}(\Omega \times Y, d x \times d \mu)^{N}=L^{p}(\Omega \times Y)^{N}$. Let $f$ be a function on $\mathbb{R}^{N} \times \mathbb{R}^{N}$ which is $\mu$-measurable and $Y$-periodic in $y$, convex in $\xi$ and satisfying, for some positive constants $c_{1}, c_{2}$, the estimate

$$
c_{1}|\xi|^{p} \leq f(y, \xi) \leq c_{2}\left(1+|\xi|^{p}\right) .
$$

Then

$$
\liminf _{\varepsilon \rightarrow 0} \int_{\Omega} f\left(\frac{x}{\varepsilon}, v_{\varepsilon}(x)\right) d \mu_{\varepsilon} \geq \int_{\Omega} \int_{Y} f(y, v(x, y)) d \mu d x .
$$

Proof. Let $f^{*}: \mathbb{R}^{N} \times \mathbb{R}^{N} \rightarrow \mathbb{R}$ be the polar of $f$ with respect to $\xi$, defined by

$$
f^{*}(y, \eta)=\sup _{\xi \in \mathbb{R}^{N}}\{\xi \cdot \eta-f(y, \xi)\} .
$$

Then $f^{*}(y, \cdot)$ is convex and the inequalities (29) imply that

$$
-c_{3}+c_{3}|\eta|^{q} \leq f^{*}(y, \eta) \leq c_{4}|\eta|^{q},
$$


for some constants $c_{3}, c_{4}$ (thus $f^{*}(y, \cdot)$ is continuous). The bipolar function $f^{* *}: \mathbb{R}^{N} \times \mathbb{R}^{N} \rightarrow \mathbb{R}$ of $f$ with respect to $\xi$, defined as

$$
f^{* *}(y, \xi)=\sup _{\eta \in \mathbb{R}^{N}}\left\{\xi \cdot \eta-f^{*}(y, \eta)\right\} .
$$

Since $f(y, \cdot)$ is convex and continuous we have that $f=f^{* *}$, see e.g. $[9$, p. 91]. From (30) this we see that (Fenchel inequality)

$\int_{\Omega} f\left(\frac{x}{\varepsilon}, v_{\varepsilon}(x)\right) d \mu_{\varepsilon} \geq \int_{\Omega} v_{\varepsilon}(x) \cdot \phi\left(x, \frac{x}{\varepsilon}\right) d \mu_{\varepsilon}-\int_{\Omega} f^{*}\left(x / \varepsilon, \phi\left(x, \frac{x}{\varepsilon}\right)\right) d \mu_{\varepsilon}$,

for any $\phi \in D^{N}$ ( $D$ defined as in (7)). By assumption $\left(v_{\varepsilon}\right)$ two-scale converges weakly. Thus we can pass to the limit in the first term in the right hand side of (32) to find that

$$
\lim _{\varepsilon \rightarrow \infty} \int_{\Omega} v_{\varepsilon}(x) \cdot \phi\left(x, \frac{x}{\varepsilon}\right) d \mu_{\varepsilon}=\int_{\Omega} \int_{Y} v(x, y) \cdot \phi(x, y) d \mu d x .
$$

Concerning the second term in the right hand side of (32) we note that $f^{*}(y, \phi(x, y))$ satisfies the conditions in Theorem 2. Hence

$$
\begin{aligned}
\liminf _{\varepsilon \rightarrow 0} \int_{\Omega} f\left(\frac{x}{\varepsilon}, v_{\varepsilon}(x)\right) d \mu_{\varepsilon} \geq & \int_{\Omega} \int_{Y} v(x, y) \cdot \phi(x, y) d \mu d x \\
& -\int_{\Omega} \int_{Y} f^{*}(y, \phi(x, y)) d \mu d x .
\end{aligned}
$$

From the growth conditions (31) it follows that the right hand side is continuous on $L^{q}(\Omega \times Y, d x \times d \mu)^{N}$ in the strong topology, see e.g. [4, p.16]. This means that the inequality (33) holds for every $\phi \in L^{q}(\Omega \times Y, d x \times d \mu)^{N}$. Let $F^{* *}(v)$ be defined as the suprenum of the right hand side of (33) over all $\phi \in L^{q}(\Omega \times Y, d x \times d \mu)^{N}$. Then by standard results concerning regularization of integral functionals, see e.g. [5, p. 270], we have that

$$
F^{* *}(v)=\int_{\Omega} \int_{Y} f^{* *}(y, v(x, y)) d \mu d x .
$$

This together with (33) and the fact that $f=f^{* *}$ gives

$$
\begin{aligned}
\liminf _{\varepsilon \rightarrow 0} \int_{\Omega} f\left(\frac{x}{\varepsilon}, v_{\varepsilon}(x)\right) d \mu_{\varepsilon} & \geq \int_{\Omega} \int_{Y} f^{* *}(y, v(x, y)) d \mu d x \\
& =\int_{\Omega} \int_{Y} f(y, v(x, y)) d \mu d x
\end{aligned}
$$

and the proof is complete. 


\section{Some special Sobolev spaces and two-scale convergence}

In this section we present some definitions and facts concerning variable Sobolev spaces. Moreover, we prove a result concerning two-scale convergence in variable Sobolev spaces, which is very important in homogenization. In the special case $p=2$ we also refer to [13].

4.1. Periodic Sobolev spaces. We define the periodic Sobolev space $W_{\text {per }}^{1, p}(Y, d \mu)$ as the closure of the set of pairs $(u, D u)$ where $u \in C_{\text {per }}^{\infty}(Y)$ in $L^{p}(Y, d \mu) \times L^{p}(Y, d \mu)^{N}$. The elements in $W_{\text {per }}^{1, p}(Y, d \mu)$ are thus pairs $\bar{u}=(u, z)$ where the second component $z$ is said to be a gradient of the first component $u$ and is denoted by $D u$. The first component in $W_{\text {per }}^{1, p}(Y, d \mu)$ can have many gradients. Each gradient $z$ corresponding to a fix first component $u$ of a pair in $W_{\text {per }}^{1, p}(Y, d \mu)$ has the form $D u+v$ where $D u$ is some gradient and $v$ is a gradient of zero. Indeed, let $D u$ be a fix gradient of $u$ and $z$ an arbitrary gradient of $u$. Then there exists $u_{n} \in C_{\mathrm{per}}^{\infty}(Y)$ and $v_{n} \in C_{\mathrm{per}}^{\infty}(Y)$ such that

$$
\begin{array}{ll}
\int_{Y}\left|u_{n}-u\right|^{p} d \mu \rightarrow 0, & \int_{Y}\left|D u_{n}-D u\right|^{p} d \mu \rightarrow 0, \\
\int_{Y}\left|v_{n}-u\right|^{p} d \mu \rightarrow 0, & \int_{Y}\left|D v_{n}-z\right|^{p} d \mu \rightarrow 0 .
\end{array}
$$

For $v_{n}-u_{n} \in C_{\mathrm{per}}^{\infty}(Y)$ we get

$$
\int_{Y}\left|v_{n}-u_{n}\right|^{p} d \mu \rightarrow 0, \quad \int_{Y}\left|D\left(v_{n}-u_{n}\right)-(z-D u)\right|^{p} d \mu \rightarrow 0 .
$$

This means that $(0, z-D u) \in W_{\text {per }}^{1, p}(Y, d \mu)$. Thus $v=z-D u$ is a gradient of zero which implies that $z=D u+(z-D u)=D u+v$. Below we give an example of the non-uniqueness of the gradient.

Example 1. Let $N=2$ and $\mu$ the one-dimensional Lebesgue measure on the interval $I=[0,1] \times\{1 / 2\}$. Fix $(u, z) \in W_{\text {per }}^{1, p}(Y, d \mu)$ then there exists $u_{n} \in C_{\text {per }}^{\infty}(Y)$ such that

$$
\begin{aligned}
\int_{Y}\left|u_{n}-u\right|^{p} d \mu & =\int_{I}\left|u_{n}-u\right|^{p} d x_{1} \rightarrow 0, \\
\int_{Y}\left|\frac{\partial u_{n}}{\partial x_{1}}-z_{1}\right|^{p} d \mu & =\int_{I}\left|\frac{\partial u_{n}}{\partial x_{1}}-z_{1}\right|^{p} d x_{1} \rightarrow 0, \\
\int_{Y}\left|\frac{\partial u_{n}}{\partial x_{2}}-z_{2}\right|^{p} d \mu & =\int_{I}\left|\frac{\partial u_{n}}{\partial x_{2}}-z_{2}\right|^{p} d x_{1} \rightarrow 0 .
\end{aligned}
$$

Thus $u$ belongs to the usual one-dimensional Sobolev space $W_{\text {per }}^{1, p}(I)$ and $z_{1}=\partial u / \partial x_{1}$, that is $D u=\left(\partial u / \partial x_{1}, z_{2}\right)$. We will now see that $z_{2}$ is not unique. Let $v_{n}=u_{n}+f\left(x_{1}\right) g\left(x_{2}\right)$, where $f$ and $g$ are smooth periodic 
functions such that $g(1 / 2)=0$ and $g^{\prime}(1 / 2)=1$. Then $v_{n} \in C_{\text {per }}^{\infty}(I)$ and

$$
\begin{aligned}
\int_{I}\left|v_{n}\left(x_{1}, 1 / 2\right)-u\left(x_{1}, 1 / 2\right)\right|^{p} d x_{1} & \rightarrow 0, \\
\int_{I}\left|\frac{\partial v_{n}}{\partial x_{1}}\left(x_{1}, 1 / 2\right)-z_{1}\right|^{p} d x_{1} & \rightarrow 0, \\
\int_{I}\left|\frac{\partial v_{n}}{\partial x_{2}}\left(x_{1}, 1 / 2\right)-\left(z_{2}-f\left(x_{1}\right)\right)\right|^{p} d x_{1} & \rightarrow 0 .
\end{aligned}
$$

Thus $\left(z_{1}, z_{2}+f\right)$ is a gradient of $u$. By density this is also true for any $f \in L^{p}(I)=L^{p}(Y, d \mu)$.

4.2. $\boldsymbol{P}$-connected measures. A measure $\mu$ is said to be $p$-connected if for each pair of the form $(u, 0) \in W_{\text {per }}^{1, p}(Y, d \mu)$ (i.e. the gradient of the first component is zero), the first component $u$ is constant $\mu$-almost everywhere or in other words if there exists $u_{n} \in C_{\text {per }}^{\infty}(Y)$ such that

$$
\int_{Y}\left|u_{n}-u\right|^{p} d \mu \rightarrow 0 \text { and } \int_{Y}\left|D u_{n}\right|^{p} d \mu \rightarrow 0,
$$

then $u$ is constant $\mu$-almost everywhere. Two important examples where the measure $\mu$ is $p$-connected are the following:

Example 2. Let the measure $\mu$ be absolutely continuous with respect to the Lebesgue measure, i.e. $d \mu=w(x) d x$. If $\int_{Y} w^{-1 /(p-1)} d x<\infty$, then $\mu$ is $p$-connected. Indeed

$$
\int_{Y}\left|D u_{n}\right| d x \leq\left(\int_{Y} w^{-1 /(p-1)} d x\right)^{(p-1) / p}\left(\int_{Y}\left|D u_{n}\right|^{p} w d x\right)^{1 / p} \rightarrow 0 .
$$

Similarly we obtain that $\int_{Y}\left|u_{n}-u\right| d x \rightarrow 0$. Thus $u$ belong to the usual Sobolev space $W^{1,1}(Y)$ and from (34) it follows that $u$ is constant a.e. with respect to the Lebesgue measure and thus a.e. with respect to $\mu$.

Example 3. Let $A$ be an open periodic set in $\mathbb{R}^{N}\left(A \subset \mathbb{R}^{N}\right.$ is periodic iff its characteristic function is periodic) and let $d \mu=w(x) d x$, where

$$
w(x)= \begin{cases}|Y \cap A|^{-1} & \text { in } A, \\ 0 & \text { outside } A .\end{cases}
$$

Then the measure $\mu$ is $p$-connected if the set $A \subset \mathbb{R}^{N}$ is connected in the usual sense (observe that this condition is not necessary). For more information see [10].

By the notation $a=\operatorname{div} b$ we mean that there exists $a \in L_{\text {per }}^{1}(Y, d \mu)$ and $b \in L_{\text {per }}^{1}(Y, d \mu)^{N}$ such that

$$
\int_{Y} a \phi d \mu=-\int_{Y} b \cdot D \phi d \mu \quad \forall \phi \in C_{\mathrm{per}}^{\infty}(Y) .
$$


The identity (35) can be equivalently rewritten as

$$
\int_{\mathbb{R}^{N}} a \phi d \mu=-\int_{\mathbb{R}^{N}} b \cdot D \phi d \mu, \quad \forall \phi \in C_{0}^{\infty}\left(\mathbb{R}^{N}\right) .
$$

We observe that each function $a$ admitting the representation $a=\operatorname{div} b$ has mean value zero, i.e. $\int_{Y} a d \mu=0$.

Theorem 9. If the measure $\mu$ is p-connected, then the set of functions $a \in L^{q}(Y, d \mu)$ which can be represented as $a=\operatorname{div} b, b \in L^{q}(Y, d \mu)^{N}$, is dense in the subspace of functions in $L^{q}(Y, d \mu)$ with mean value zero.

Proof. Let $A$ be the set of functions $a \in L^{q}(Y, d \mu)$ which can be represented as $a=\operatorname{div} b, b \in L^{q}(Y, d \mu)^{N}$. Define the annihilator $A^{\perp}$ as the set of functions $f \in L^{p}(Y, d \mu)$ such that $\int_{Y} f d \mu=0$ and $\int_{Y} f a d \mu=0$ for any $a \in A$. If we prove that $A^{\perp}=\{0\}$ it follows by Theorem 4.7 in [8] that $A$ is dense in $L^{q}(Y, d \mu)$ and we are done. Fix $f \in A^{\perp}$ and consider the periodic problem: Find $(u, D u) \in W_{\text {per }}^{1, p}(Y, d \mu)$ such that

$$
\int_{Y}\left(|D u|^{p-2} D u \cdot D \phi+|u|^{p-2} u \phi\right) d \mu=\int_{Y}|f|^{p-2} f \phi d \mu, \quad \forall \phi \in C_{\mathrm{per}}^{\infty}(Y),
$$

(as test function we may chose $\left.(\phi, D \phi) \in W_{\text {per }}^{1, p}(Y, d \mu)\right)$. This problem is known to be solvable. Obviously $|f|^{p-2} f-|u|^{p-2} u \in A$ and since $f \in A^{\perp}$ we have that $\int_{Y}\left(|f|^{p}-|u|^{p-2} u f\right) d \mu=0$. The Hölder inequality implies that

$$
\int_{Y}|f|^{p} d \mu=\int_{Y}|u|^{p-2} u f d \mu \leq\left(\int_{Y}|u|^{(p-1) q} d \mu\right)^{1 / q}\left(\int_{Y}|f|^{p} d \mu\right)^{1 / p},
$$

i.e. that

$$
\int_{Y}|f|^{p} d \mu \leq \int_{Y}|u|^{p} d \mu .
$$

By choosing $\phi=u$ in (37) we obtain

$$
\int_{Y}\left(|D u|^{p}+|u|^{p}\right) d \mu=\int_{Y}|f|^{p-2} f u d \mu \leq\left(\int_{Y}|f|^{p} d \mu\right)^{1 / q}\left(\int_{Y}|u|^{p} d \mu\right)^{1 / p}
$$

and hence

$$
\int_{Y}|u|^{p} d \mu \leq \int_{Y}|f|^{p} d \mu \text { and } \int_{Y}\left(|D u|^{p}+|u|^{p}\right) d \mu \leq \int_{Y}|f|^{p} d \mu .
$$

This together with (38) gives

$$
\int_{Y}|u|^{p} d \mu=\int_{Y}|f|^{p} d \mu \quad \text { and } \quad \int_{Y}|D u|^{p} d \mu=0 .
$$

By the $p$-connectedness of $\mu$ it follows that $u$ is constant $\mu$-almost everywhere. This fact together with equation (37) implies that $f$ is constant 
$\mu$-almost everywhere and since $\int_{Y} f d \mu=0$ we must have that $f=0$. The proof is complete.

4.3. Degenerate measures. We define the space $V_{\text {pot }}^{p}$ of potential vectors as the closure of the set $\left\{D \phi: \phi \in C_{\mathrm{per}}^{\infty}(Y)\right\}$ in $L^{p}(Y, d \mu)^{N}$. A vector $b \in L^{q}(Y, d \mu)^{N}$ is said to be solenoidal if $b \in\left(V_{\text {pot }}^{p}\right)^{\perp}$, i.e.

$$
\int_{Y} b \cdot D \phi d \mu=0, \quad \forall \phi \in C_{\mathrm{per}}^{\infty}(Y) .
$$

The so defined set of solenoidal vectors is denoted by $V_{\mathrm{sol}}^{q}$. Since every norm-closed subspace of $L^{p}(Y, d \mu)^{N}$ is the annihilator of its annihilator (see $[8$, p. 96]) we have

$$
V_{\mathrm{sol}}^{q}=\left(V_{\mathrm{pot}}^{p}\right)^{\perp} \quad \text { and } \quad V_{\text {pot }}^{p}=\left(V_{\mathrm{sol}}^{q}\right)^{\perp} .
$$

For the Hilbert space case, $p=2$, we have the orthogonal decomposition

$$
L^{2}(Y, d \mu)^{N}=V_{\text {sol }}^{2} \oplus V_{\text {pot }}^{2} .
$$

Let $L^{p}\left(\Omega, V_{\text {pot }}^{p}\right)$ be defined as the closure in $L^{p}(\Omega \times Y)$ of the linear span of vectors $f(x) D \phi(y)$, where $f \in C_{0}^{\infty}(\Omega)$ and $\phi \in C_{\text {per }}^{\infty}(Y)$. Correspondingly, $L^{q}\left(\Omega, V_{\mathrm{sol}}^{q}\right)$ is defined as closure in $L^{q}(\Omega \times Y)$ of the linear span of vectors $f(x) b(y)$, where $f \in C_{0}^{\infty}(\Omega)$ and $b \in V_{\mathrm{sol}}^{q}$. By (39) it follows that

$$
L^{q}\left(\Omega, V_{\mathrm{sol}}^{q}\right)^{\perp}=L^{p}\left(\Omega, V_{\mathrm{pot}}^{p}\right) \quad \text { and } \quad L^{p}\left(\Omega, V_{\mathrm{pot}}^{p}\right)^{\perp}=L^{q}\left(\Omega, V_{\mathrm{sol}}^{q}\right) .
$$

For some measures a non-zero constant vector may be a potential vector. Let $E$ be the subspace of $\mathbb{R}^{N}$ of such potential vectors and $E^{\perp}$ its orthogonal complement. We say that the measure $\mu$ is non-degenerate if $E=\{0\}$.

Example 4. Let $N=2$ and let $\mu$ be the one-dimensional Lebesgue measure on the interval $I=[0,1] \times\{1 / 2\}$. Let $g=g\left(x_{2}\right)$ be smooth periodic function such that $g(1 / 2)=0$ and $g^{\prime}(1 / 2)=1$. Let be defined as $v_{n}\left(x_{1}, x_{2}\right)=k g\left(x_{2}\right)$. Then $v_{n} \in C_{\mathrm{per}}^{\infty}(Y)$ and

$$
\begin{aligned}
\int_{Y}\left|\frac{\partial v_{n}}{\partial x_{1}}\right|^{p} d \mu & =\int_{I}\left|\frac{\partial v_{n}}{\partial x_{1}}\left(x_{1}, 1 / 2\right)\right|^{p} d x_{1} \rightarrow 0, \\
\int_{Y}\left|\frac{\partial v_{n}}{\partial x_{2}}-k\right|^{p} d \mu & =\int_{I}\left|\frac{\partial v_{n}}{\partial x_{2}}\left(x_{1}, 1 / 2\right)-k\right|^{p} d x_{1} \rightarrow 0 .
\end{aligned}
$$

Thus the constant vector $(0, k) \in V_{\text {pot }}^{p}$.

Theorem 10. If $m: \mathbb{R}^{N} \rightarrow \mathbb{R}$ is given by

$$
m(\xi)=\min _{v \in V_{\text {pot }}^{p}} \int_{Y}|\xi+v|^{p} d \mu .
$$

Then

1. $m$ is convex. 
2. $m(\xi+\eta)=m(\xi)$ for any $\xi \in \mathbb{R}^{N}$ and $\eta \in E$.

3. There exists a constant $c>0$ such that $m(\xi) \geq c|\xi|^{p}$ for $\xi \in E^{\perp}$, i.e. $m$ is coercive on $E^{\perp}$.

Proof. 1. Let $\xi_{1}$ and $\xi_{2}$ be two arbitrary vectors in $\mathbb{R}^{N}$ and let $v_{1}$ and $v_{2}$ be the corresponding minimizers of (41). Then, for $0 \leq \alpha \leq 1$, the convexity of the function $|\cdot|^{p}$ implies that

$$
\begin{aligned}
m\left(\alpha \xi_{1}+(1-\alpha) \xi_{2}\right) & =\min _{v \in V_{\mathrm{pot}}^{p}} \int_{Y}\left|\alpha \xi_{1}+(1-\alpha) \xi_{2}+v\right|^{p} d \mu \\
& \leq \int_{Y}\left|\alpha \xi_{1}+(1-\alpha) \xi_{2}+\alpha v_{1}+(1-\alpha) v_{2}\right|^{p} d \mu \\
& \leq \int_{Y} \alpha\left|\xi_{1}+v_{1}\right|^{p}+(1-\alpha)\left|\xi_{2}+v_{2}\right|^{p} d \mu \\
& =\alpha m\left(\xi_{1}\right)+(1-\alpha) m\left(\xi_{2}\right),
\end{aligned}
$$

which proves that $m$ is convex.

2 . Let $t \in \mathbb{R}$. The following estimates are valid

$$
0 \leq m(\xi+t \eta) \leq \min _{v \in E} \int_{Y}|\xi+t \eta+v|^{p} d \mu \leq|\xi|^{p} .
$$

The convexity of $m$ implies that the function $f(t)=m(\xi+t \eta)$ is convex and by (42) $f$ is bounded between two constants. Thus $f$ is constant, which gives the desired result

$$
m(\xi+\eta)=m(\xi) \text { for any } \xi \in \mathbb{R}^{N} \text { and } \eta \in E .
$$

3. We have that $m(\xi)>0$ for $\xi \notin E$. The homogeneity implies that

$$
m(\xi)=\min _{v \in V_{\mathrm{pot}}^{p}} \int_{Y}|\xi+v|^{p} d \mu=|\xi|^{p} \min _{v \in V_{\mathrm{pot}}^{2}} \int_{Y}|\eta+v|^{p} d \mu,
$$

where $\eta=|\xi|^{-1} \xi$. The set $\left\{\eta: \eta \in E^{\perp}\right\}$ is compact. Hence the continuous function $\beta$ defined as

$$
\beta(\eta) \stackrel{\text { def }}{=} \min _{v \in V_{\text {pot }}^{p}} \int_{Y}|\eta+v|^{p} d \mu
$$

attains its minimum when $\eta$ rages over $\left\{\eta: \eta \in E^{\perp}\right\}$. Denote this strictly positive minimum by $c$. Then it follows from (43) that

$$
m(\xi) \geq c|\xi|^{p} \text { for } \xi \in E^{\perp},
$$

i.e. $m$ is coercive on $E^{\perp}$.

Theorem 11. $V_{\text {sol }}^{q}$ has the following properties:

1. The mean value of a vector $b \in V_{\text {sol }}^{q}$ belong to $E^{\perp}$.

2. Each vector in $E^{\perp}$ is the mean value of a vector $b \in V_{\text {sol }}^{q}$. 
Proof. 1. Let $b \in V_{\mathrm{sol}}^{q}$, which by definition means that

$$
\int_{Y} b \cdot v d \mu=0, \quad \forall v \in V_{\text {pot }}^{p} .
$$

This implies that

$$
\int_{Y} b \cdot \xi d \mu=\int_{Y} b d \mu \cdot \xi=0, \quad \forall \xi \in E .
$$

Thus the mean value of $b$ belongs to $E^{\perp}$.

2. The minimum problem (41) has a unique solution, which satisfies the Euler equation: Find $v \in V_{\mathrm{pot}}^{p}$ such that

$$
\int_{Y}|\xi+v|^{p-2}(\xi+v) \cdot w d \mu=0, \quad \forall w \in V_{\mathrm{pot}}^{p} .
$$

Let $v$ and $\widehat{v}$ be the solutions corresponding to $\xi \in \mathbb{R}^{N}$ and $\widehat{\xi} \in \mathbb{R}^{N}$, respectively. Then, by the convexity of $|\cdot|^{p}$,

$$
\begin{aligned}
m(\widehat{\xi})-m(\xi) & =\int_{Y}|\widehat{\xi}+\widehat{v}|^{p}-|\xi+v|^{p} d \mu \\
& \geq \int_{Y} p|\xi+v|^{p-2}(\xi+v) \cdot(\widehat{\xi}+\widehat{v}-\xi-v) d \mu .
\end{aligned}
$$

This together with the fact that $v$ solves the Euler equation (44) implies that

$$
m(\widehat{\xi})-m(\xi) \geq p \int_{Y}|\xi+v|^{p-2}(\xi+v) d \mu \cdot(\widehat{\xi}-\xi) .
$$

By the convexity of $m$ it follows that

$$
\operatorname{Dm}(\xi)=p \int_{Y}|\xi+v|^{p-2}(\xi+v) d \mu .
$$

From (44) it is clear that $|\xi+v|^{p-2}(\xi+v) \in V_{\text {sol }}^{q}$ and by the first part of the theorem we have that it's mean value belong to $E^{\perp}$, i.e. $\operatorname{Dm}(\xi) \in E^{\perp}$. Since $m$ is coercive on $E^{\perp}$ (see Theorem 10) it holds that for every $\eta \in E^{\perp}$ there exists a $\xi$ such $\operatorname{Dm}(\xi)=\eta$.

\subsection{Two-scale convergence in variable Sobolev spaces.}

Theorem 12. Let $\mu$ be a p-connected non-degenerate measure. Assume that $\left(u_{\varepsilon}\right)$ is a sequence in $C_{0}^{\infty}(\Omega)$ such that $u_{\varepsilon}(x) \stackrel{2}{\rightarrow} u(x, y)$ and $D u_{\varepsilon}(x) \stackrel{2}{\rightarrow}$ $z(x, y)$. Then the weak two-scale limit $u$ is independent of $y$ and belong to $W_{0}^{1, p}(\Omega)$, i.e. $u(x, y)=u(x) \in W_{0}^{1, p}(\Omega)$. Moreover, $z(x, y)=D u(x)+$ $v(x, y)$, where $v \in L^{p}\left(\Omega, V_{\text {pot }}^{p}\right)$. 
Proof. Let $\psi \in C_{0}^{\infty}(\Omega)$ and chose $b \in L_{\mathrm{per}}^{q}(Y, d \mu)^{N}$ and $a \in L_{\mathrm{per}}^{q}(Y, d \mu)$ such that $a=\operatorname{div} b$. The identity (36) implies that

$$
\varepsilon \int_{\Omega} D \varphi(x) \cdot b\left(\frac{x}{\varepsilon}\right) d \mu_{\varepsilon}=-\int_{\Omega} \varphi(x) a\left(\frac{x}{\varepsilon}\right) d \mu_{\varepsilon}, \quad \forall \varphi \in C_{0}^{\infty}(\Omega) .
$$

Partial differentiation gives

$$
\begin{aligned}
\varepsilon \int_{\Omega} D\left(\psi(x) u_{\varepsilon}(x)\right) \cdot b\left(\frac{x}{\varepsilon}\right) d \mu_{\varepsilon}= & \varepsilon \int_{\Omega} u_{\varepsilon}(x) D \psi(x) \cdot b\left(\frac{x}{\varepsilon}\right) d \mu_{\varepsilon} \\
& +\varepsilon \int_{\Omega} \psi(x) D u_{\varepsilon}(x) \cdot b\left(\frac{x}{\varepsilon}\right) d \mu_{\varepsilon} .
\end{aligned}
$$

This together with (45) means that

$$
\begin{aligned}
-\int_{\Omega} \psi(x) u_{\varepsilon}(x) a\left(\frac{x}{\varepsilon}\right) d \mu_{\varepsilon}= & \varepsilon \int_{\Omega} u_{\varepsilon}(x) D \psi(x) \cdot b\left(\frac{x}{\varepsilon}\right) d \mu_{\varepsilon} \\
& +\varepsilon \int_{\Omega} \psi(x) D u_{\varepsilon}(x) \cdot b\left(\frac{x}{\varepsilon}\right) d \mu_{\varepsilon} .
\end{aligned}
$$

The right hand side tends to zero as $\varepsilon \rightarrow 0$ since $\left(u_{\varepsilon}\right)$ and $\left(D u_{\varepsilon}\right)$ two-scale converge weakly by assumption. Thus by passing to the limit we obtain

$$
\int_{\Omega} \int_{Y} \psi(x) u(x, y) a(y) d \mu d x=0 .
$$

We note that $\int_{Y} a(y) d \mu=0$ and by Theorem 9 the set of functions $a \in L^{q}(Y, d \mu)$ which can be represented as $a=\operatorname{div} b, b \in L^{q}(Y, d \mu)^{N}$, is dense in the subspace of functions in $L^{q}(Y, d \mu)$ with mean value zero. Hence $u$ is independent of $y$.

Next we prove that $u \in W_{0}^{1, p}(\Omega)$ and that $z(x, y)=D u(x)+v(x, y)$, where $v \in L^{p}\left(\Omega, V_{\text {pot }}^{p}\right)$. For $b \in V_{\text {sol }}^{q}$ with $\int_{Y} b d \mu=\eta=\left(\eta_{1}, \ldots, \eta_{N}\right)$ and $\phi \in C^{\infty}(\bar{\Omega})$ we have the identity

$$
0=\int_{\Omega} D\left(\phi u_{\varepsilon}\right) \cdot b(x / \varepsilon) d \mu_{\varepsilon}=\int_{\Omega} u_{\varepsilon} D \phi \cdot b(x / \varepsilon)+D u_{\varepsilon} \cdot \phi b(x / \varepsilon) d \mu_{\varepsilon} .
$$

By passing to the limit in the weak two-scale sense we get

$$
0=\int_{\Omega} \int_{Y} u(x) D \phi(x) \cdot b(y) d \mu(y) d x+\int_{\Omega} \int_{Y} z(x, y) \cdot \phi(x) b(y) d \mu(y) d x .
$$

Thus

$$
\int_{\Omega} u(x) D \phi(x) \cdot \eta d x=-\int_{\Omega} v_{b}(x) \phi(x) d x \quad \forall \phi \in C^{\infty}(\bar{\Omega}),
$$

where $v_{b}(x)=\int_{Y} z(x, y) \cdot b(y) d \mu(y)$. Since $\mu$ is non-degenerate there exists $b^{i} \in V_{\mathrm{sol}}^{q}$ such that

$$
\int_{\Omega} u D_{i} \phi d x=-\int_{\Omega} v_{b^{i}} \phi d x \quad \forall \phi \in C^{\infty}(\bar{\Omega}), \quad i=1, \ldots, N .
$$


Thus the distributional partial derivatives $D_{i} u=v_{b^{i}}$ of $u$ are in $L^{p}(\Omega)$, that is $u \in W^{1, p}(\Omega)$. Moreover, equation (47) together with the integration by parts formula gives that $u \in W_{0}^{1, p}(\Omega)$ for $\Omega$ with Lipschitz property. The equality (46) can now be written as

$$
\begin{aligned}
\int_{Y} \int_{\Omega} z(x, y) \cdot \phi(x) b(y) d x d \mu(y) & =-\int_{Y} \int_{\Omega} u(x) D \phi(x) \cdot b(y) d x d \mu(y) \\
& =\int_{Y} \int_{\Omega} \phi(x) D u(x) \cdot b(y) d x d \mu(y) .
\end{aligned}
$$

Hence

$$
\int_{Y} \int_{\Omega}[z(x, y)-D u(x)] \cdot \phi(x) b(y) d x d \mu(y)=0 .
$$

Since $L^{q}\left(\Omega, V_{\mathrm{sol}}^{q}\right)$ is defined as closure in $L^{q}(\Omega \times Y)$ of the linear span of vectors $f(x) b(y)$, where $f \in C_{0}^{\infty}(\Omega)$ and $b \in V_{\text {sol }}^{q}$ we obtain that

$$
\int_{Y} \int_{\Omega}[z(x, y)-D u(x)] \cdot w(x, y) d x d \mu(y)=0, \quad \forall w \in L^{q}\left(\Omega, V_{\mathrm{sol}}^{q}\right) .
$$

From (40) we see that $[z(x, y)-D u(x)]$ belong to $L^{p}\left(\Omega, V_{\text {pot }}^{p}\right)$ or in other words $z(x, y)=D u(x)+v(x, y)$, where $v \in L^{p}\left(\Omega, V_{\text {pot }}^{p}\right)$.

We remark that it is possible to derive a similar result as in Theorem 12 for degenerate measures, see [13] for $p=2$. However this is beyond the scope of this paper. So far we have considered sequences $\left(u_{\varepsilon}\right)$ in $C_{0}^{\infty}(\Omega)$. However, everything holds true also for sequences $\left(u_{\varepsilon}\right)$ in the variable Sobolev space $W_{0}^{1, p}\left(\Omega, d \mu_{\varepsilon}\right)$, where $W_{0}^{1, p}\left(\Omega, d \mu_{\varepsilon}\right)$ is defined as the closure of the set of pairs $(u, D u)$ where $u \in C_{0}^{\infty}(\Omega)$ in $L^{p}\left(Y, d \mu_{\varepsilon}\right) \times L^{p}\left(Y, d \mu_{\varepsilon}\right)^{N}$.

\section{Monotone operators and two-scale convergence}

In this section a class of monotone operators are defined (the corresponding homogenization problem is analyzed in the next section). It is proved that the related operator equations have unique solutions. Moreover, some results concerning two-scale convergence and monotonicity are proved.

5.1. A class of monotone operators. By definition $W_{0}^{1, p}\left(\Omega, d \mu_{\varepsilon}\right)$ is a closed subspace of $L^{p}\left(Y, d \mu_{\varepsilon}\right) \times L^{p}\left(Y, d \mu_{\varepsilon}\right)^{N}$. Since closed subspaces of reflexive Banach spaces are reflexive we have that $W_{0}^{1, p}\left(\Omega, d \mu_{\varepsilon}\right)$ is reflexive. Let $T$ be an operator from $W_{0}^{1, p}\left(\Omega, d \mu_{\varepsilon}\right)$ into its dual $W_{0}^{1, p}\left(\Omega, d \mu_{\varepsilon}\right)^{*}$ and let $\langle\cdot, \cdot\rangle$ denote the dual pairing between $W_{0}^{1, p}\left(\Omega, d \mu_{\varepsilon}\right)$ and $W_{0}^{1, p}\left(\Omega, d \mu_{\varepsilon}\right)^{*}$. We recall the following definitions:

- $T$ is called monotone if

$$
\langle T \bar{u}-T \bar{v}, \bar{u}-\bar{v}\rangle \geq 0 \quad \forall \bar{u}, \bar{v} \in W_{0}^{1, p}\left(\Omega, d \mu_{\varepsilon}\right) .
$$


- $T$ is called strictly monotone if

$$
\langle T \bar{u}-T \bar{v}, \bar{u}-\bar{v}\rangle>0 \text { whenever } \bar{u} \neq \bar{v} .
$$

- $T$ is called hemicontinuous if

$$
\lim _{t \rightarrow 0}\langle T(\bar{u}+t \bar{v}), \bar{w}\rangle=\langle T \bar{u}, \bar{w}\rangle,
$$

for all $\bar{u}, \bar{v}, \bar{w} \in W_{0}^{1, p}\left(\Omega, d \mu_{\varepsilon}\right)$.

- $T$ is called coercive if

$$
\lim _{\|\bar{u}\| \rightarrow \infty} \frac{\langle T \bar{u}, \bar{u}\rangle}{\|\bar{u}\|}=+\infty
$$

The Browder-Minty theorem states that if $T$ is strictly monotone, hemicontinuous and coercive, then the operator equation

$$
T \bar{u}=g
$$

has a unique solution $\bar{u} \in W_{0}^{1, p}\left(\Omega, d \mu_{\varepsilon}\right)$ for every $g \in W_{0}^{1, p}\left(\Omega, d \mu_{\varepsilon}\right)^{*}$.

Let $a: \mathbb{R}^{N} \times \mathbb{R}^{N} \rightarrow \mathbb{R}^{N}$ be a function such that $a(\cdot, \xi)$ is $Y$-periodic and $\mu$ measurable for every $\xi \in \mathbb{R}^{N}$. Moreover, assume that there exists constants $c_{1}, c_{2}>0$ and two more constants $\alpha$ and $\beta$, with $0 \leq \alpha \leq \min \{1, p-1\}$ and $\max \{p, 2\} \leq \beta<\infty$ such that $a$ satisfies the following continuity and monotonicity assumptions:

$$
\begin{gathered}
a(y, 0)=0 \\
\left|a\left(y, \xi_{1}\right)-a\left(y, \xi_{2}\right)\right| \leq c_{1}\left(1+\left|\xi_{1}\right|+\left|\xi_{2}\right|\right)^{p-1-\alpha}\left|\xi_{1}-\xi_{2}\right|^{\alpha}, \\
\left(a\left(y, \xi_{1}\right)-a\left(y, \xi_{2}\right)\right) \cdot\left(\xi_{1}-\xi_{2}\right) \geq c_{2}\left(1+\left|\xi_{1}\right|+\left|\xi_{2}\right|\right)^{p-\beta}\left|\xi_{1}-\xi_{2}\right|^{\beta},
\end{gathered}
$$

for a.e. $y \in \mathbb{R}^{N}$ and any $\xi_{1}, \xi_{2} \in \mathbb{R}^{N}$. A direct consequence of (48)-(50) is that there exists constants $c_{3}, c_{4}>0$ such that

$$
|a(x, \xi)| \leq c_{3}\left(1+|\xi|^{p-1}\right) \quad \text { and } \quad|\xi|^{p} \leq c_{4}(1+a(x, \xi) \cdot \xi)
$$

Fix $\varepsilon>0$ and consider the equation

$$
(52)-\operatorname{div}\left(a\left(x / \varepsilon, D u_{\varepsilon}\right)\right)+\lambda\left|u_{\varepsilon}\right|^{p-2} u_{\varepsilon}=f_{\varepsilon}, \quad\left(u_{\varepsilon}, D u_{\varepsilon}\right) \in W_{0}^{1, p}\left(\Omega, d \mu_{\varepsilon}\right),
$$

where $f_{\varepsilon} \in L^{q}\left(\Omega, d \mu_{\varepsilon}\right)$ and $\lambda>0$. By definition $\left(u_{\varepsilon}, D u_{\varepsilon}\right) \in W_{0}^{1, p}\left(\Omega, d \mu_{\varepsilon}\right)$ is a solution of $(52)$ if the following integral identity holds:

$$
\int_{\Omega} a\left(x / \varepsilon, D u_{\varepsilon}\right) \cdot D \phi+\lambda\left|u_{\varepsilon}\right|^{p-2} u_{\varepsilon} \phi d \mu_{\varepsilon}=\int_{\Omega} f_{\varepsilon} \phi d \mu_{\varepsilon}, \quad \forall \phi \in C_{0}^{\infty}(\Omega) .
$$

We remark that by density we have an equivalent definition of a solution if the test functions $\phi \in C_{0}^{\infty}(\Omega)$ in (53) is replaced by $\left(\phi, \phi_{1}\right) \in W_{0}^{1, p}\left(\Omega, d \mu_{\varepsilon}\right)$.

Theorem 13. The equation (52) has a unique solution. 
Proof. Let $T: W_{0}^{1, p}\left(\Omega, d \mu_{\varepsilon}\right) \rightarrow W_{0}^{1, p}\left(\Omega, d \mu_{\varepsilon}\right)^{*}$ and $g \in W_{0}^{1, p}\left(\Omega, d \mu_{\varepsilon}\right)^{*}$ be defined as

$$
\begin{aligned}
\left\langle T\left(u, u_{1}\right),\left(\phi, \phi_{1}\right)\right\rangle & \stackrel{\text { def }}{=} \int_{\Omega} a\left(x / \varepsilon, u_{1}\right) \cdot \phi_{1}+\lambda|u|^{p-2} u \phi d \mu_{\varepsilon} \\
\left\langle g,\left(\phi, \phi_{1}\right)\right\rangle & \stackrel{\text { def }}{=} \int_{\Omega} f_{\varepsilon} \phi d \mu_{\varepsilon} .
\end{aligned}
$$

Then the equation (52) is equivalent to the operator equation

$$
T\left(u, u_{1}\right)=g, \quad\left(u, u_{1}\right) \in W_{0}^{1, p}\left(\Omega, d \mu_{\varepsilon}\right) .
$$

To be able to use the Browder-Minty theorem we have to show that $T$ is strictly monotone, hemicontinuous and coercive.

(i) (Strictly monotone) By (50) we get

$$
\begin{aligned}
& \left\langle T\left(u, u_{1}\right)-T\left(v, v_{1}\right),\left(u, u_{1}\right)-\left(v, v_{1}\right)\right\rangle \\
& \geq \quad c_{2} \int_{\Omega}\left(1+\left|u_{1}\right|+\left|v_{1}\right|\right)^{p-\beta}\left|u_{1}-u_{2}\right|^{\beta} d \mu_{\varepsilon} \\
& \quad+\lambda \int_{\Omega}\left[|u|^{p-2} u-|v|^{p-2} v\right](u-v) d \mu_{\varepsilon} .
\end{aligned}
$$

First we consider the case $1<p \leq 2$. In view of the reversed Hölder inequality (with the dual exponents $0<p / \beta<1$ and $p /(p-\beta)$ ) and the fact that there exists a positive constant $c$ such that $\left[|u|^{p-2} u-|v|^{p-2} v\right](u-v) \geq$ $c(|u|+|v|)^{p-2}|u-v|^{2}$ it follows that

$$
\begin{aligned}
& \left\langle T\left(u, u_{1}\right)-T\left(v, v_{1}\right),\left(u, u_{1}\right)-\left(v, v_{1}\right)\right\rangle \\
& \geq c_{2}\left(\int_{\Omega}\left(1+\left|u_{1}\right|+\left|v_{1}\right|\right)^{p} d \mu_{\varepsilon}\right)^{(p-\beta) / p}\left(\int_{\Omega}\left|u_{1}-u_{2}\right|^{p} d \mu_{\varepsilon}\right)^{\beta / p} \\
& \quad+\lambda c \int_{\Omega}(|u|+|v|)^{p-2}|u-v|^{2} d \mu_{\varepsilon} .
\end{aligned}
$$

By using the reversed Hölder inequality (with the dual exponents $p / 2<1$ and $p /(p-2))$ on the second term we obtain

$$
\begin{aligned}
& \left\langle T\left(u, u_{1}\right)-T\left(v, v_{1}\right),\left(u, u_{1}\right)-\left(v, v_{1}\right)\right\rangle \\
& \geq c_{2}\left(\int_{\Omega}\left(1+\left|u_{1}\right|+\left|v_{1}\right|\right)^{p} d \mu_{\varepsilon}\right)^{(p-\beta) / p}\left(\int_{\Omega}\left|u_{1}-u_{2}\right|^{p} d \mu_{\varepsilon}\right)^{\beta / p} \\
& \quad+\lambda c\left(\int_{\Omega}(|u|+|v|)^{p} d \mu_{\varepsilon}\right)^{(p-2) / p}\left(\int_{\Omega}|u-v|^{p} d \mu_{\varepsilon}\right)^{2 / p} .
\end{aligned}
$$

This shows that $T$ is strictly monotone. Let us now consider the case $2 \leq p<\infty$. The reversed Hölder inequality (with the dual exponents 
$0<p / \beta<1$ and $p /(p-\beta))$ and the fact that there exists a positive constant $c$ such that $\left[|u|^{p-2} u-|v|^{p-2} v\right](u-v) \geq c|u-v|^{p}$ it follows that

$$
\begin{aligned}
& \left\langle T\left(u, u_{1}\right)-T\left(v, v_{1}\right),\left(u, u_{1}\right)-\left(v, v_{1}\right)\right\rangle \\
& \geq c_{2}\left(\int_{\Omega}\left(1+\left|u_{1}\right|+\left|v_{1}\right|\right)^{p} d \mu_{\varepsilon}\right)^{(p-\beta) / p}\left(\int_{\Omega}\left|u_{1}-u_{2}\right|^{p} d \mu_{\varepsilon}\right)^{\beta / p} \\
& \quad+\lambda c \int_{\Omega}|u-v|^{p} d \mu_{\varepsilon} .
\end{aligned}
$$

From this we see that $T$ is strictly monotone.

(ii) (Hemicontinuous) First we consider the case $1<p<2$. By (49) and the fact that for real numbers $a$ and $b$ there exists a constant $c>0$ such that $\left.|| a\right|^{p-2} a-|b|^{p-2} b|\leq c| a-\left.b\right|^{p-1}$ we get that

$$
\begin{aligned}
& \left|\left\langle T\left(u, u_{1}\right)+t\left(v, v_{1}\right)\right)-T\left(u, u_{1}\right),\left(w, w_{1}\right)\right\rangle \mid \\
& \leq \int_{\Omega}\left|\left[a\left(\frac{x}{\varepsilon}, u_{1}+t v_{1}\right)-a\left(\frac{x}{\varepsilon}, u_{1}\right)\right]\right|\left|w_{1}\right| d \mu_{\varepsilon} \\
& \quad+\lambda \int_{\Omega}|| u+\left.t v\right|^{p-2}(u+t v)-|u|^{p-2} u|| w \mid d \mu_{\varepsilon} \\
& \leq c_{1}|t|^{\alpha} \int_{\Omega}\left(1+\left|u_{1}+t v_{1}\right|+\left|u_{1}\right|\right)^{p-1-\alpha}\left|v_{1}\right|^{\alpha}\left|w_{1}\right| d \mu_{\varepsilon} \\
& \quad+\lambda c \int_{\Omega}|t v|^{p-1}|w| d \mu_{\varepsilon} \\
& \leq c_{1}|t|^{\alpha} \int_{\Omega}\left(1+\left|u_{1}+t v_{1}\right|+\left|u_{1}\right|+\left|v_{1}\right|\right)^{p-1}\left|w_{1}\right| d \mu_{\varepsilon} \\
& \quad+\lambda c|t|^{p-1} \int_{\Omega}|v|^{p-1}|w| d \mu_{\varepsilon} .
\end{aligned}
$$

By applying the Hölder inequality we obtain

$$
\begin{aligned}
& \left|\left\langle T\left(\left(u, u_{1}\right)+t\left(v, v_{1}\right)\right)-T\left(u, u_{1}\right),\left(w, w_{1}\right)\right\rangle\right| \\
& \leq c_{1}|t|^{\alpha}\left(\int_{\Omega}\left(1+\left|u_{1}+t v_{1}\right|+\left|u_{1}\right|+\left|v_{1}\right|\right)^{p} d \mu_{\varepsilon}\right)^{1 / q}\left(\int_{\Omega}\left|w_{1}\right|^{p} d \mu_{\varepsilon}\right)^{1 / p} \\
& \quad+\lambda c|t|^{p-1}\left(\int_{\Omega}|v|^{p} d \mu_{\varepsilon}\right)^{1 / q}\left(\int_{\Omega}|w|^{p} d \mu_{\varepsilon}\right)^{1111 / p} .
\end{aligned}
$$

Since all the integrals are bounded we have that

$$
\lim _{t \rightarrow 0}\left|\left\langle T\left(\left(u, u_{1}\right)+t\left(v, v_{1}\right)\right)-T\left(u, u_{1}\right),\left(w, w_{1}\right)\right\rangle\right|=0,
$$

which by definition means that $T$ is hemicontinuous. Let us now consider the case $2 \leq p<\infty$. By the same arguments as above with the inequality for real numbers replaced by that for real numbers $a$ and $b$ there exists a 
constant $c>0$ such that $\left.|| a\right|^{p-2} a-|b|^{p-2} b\left|\leq c[|a|+|b|]^{p-2}\right| a-b \mid$ we get that

$$
\begin{aligned}
& \left|\left\langle T\left(\left(u, u_{1}\right)+t\left(v, v_{1}\right)\right)-T\left(u, u_{1}\right),\left(w, w_{1}\right)\right\rangle\right| \\
& \leq c_{1}|t|^{\alpha} \int_{\Omega}\left(1+\left|u_{1}+t v_{1}\right|+\left|u_{1}\right|+\left|v_{1}\right|\right)^{p-1}\left|w_{1}\right| d \mu_{\varepsilon} \\
& \quad+\lambda c|t| \int_{\Omega}[|u+t v|+|u|]^{p-2}|v||w| d \mu_{\varepsilon} .
\end{aligned}
$$

Hölder's inequality applied to the right hand side gives

$$
\begin{aligned}
& \left|\left\langle T\left(\left(u, u_{1}\right)+t\left(v, v_{1}\right)\right)-T\left(u, u_{1}\right),\left(w, w_{1}\right)\right\rangle\right| \\
& \leq c_{1}|t|^{\alpha}\left(\int_{\Omega}\left(1+\left|u_{1}+t v_{1}\right|+\left|u_{1}\right|+\left|v_{1}\right|\right)^{p} d \mu_{\varepsilon}\right)^{1 / q}\left(\int_{\Omega}\left|w_{1}\right|^{p} d \mu_{\varepsilon}\right)^{1 / p} \\
& \quad+\lambda c|t|\left[\int_{\Omega}[|u+t v|+|u|]^{p} d \mu_{\varepsilon}\right]^{(p-2) / p}\left[\int_{\Omega}|v|^{p} d \mu_{\varepsilon}\right]^{1 / p} \\
& \quad \times\left[\int_{\Omega}|w|^{p} d \mu_{\varepsilon}\right]^{1 / p} .
\end{aligned}
$$

Since all the integrals are bounded we have that

$$
\lim _{t \rightarrow 0}\left|\left\langle T\left(\left(u, u_{1}\right)+t\left(v, v_{1}\right)\right)-T\left(u, u_{1}\right),\left(w, w_{1}\right)\right\rangle\right|=0 .
$$

Thus $T$ is hemicontinuous.

(iii) (Coercive) By (48) and (50) we get that

$$
\left\langle T\left(u, u_{1}\right),\left(u, u_{1}\right)\right\rangle \geq c_{2} \int_{\Omega}\left(1+\left|u_{1}\right|\right)^{p-\beta}\left|u_{1}\right|^{\beta} d \mu_{\varepsilon}+\lambda \int_{\Omega}|u|^{p} d \mu_{\varepsilon} .
$$

The reversed Hölder inequality (with the dual exponents $p /(p-\beta)$ and $0<p / \beta<1)$ gives that

$$
\begin{aligned}
\left\langle T\left(u, u_{1}\right),\left(u, u_{1}\right)\right\rangle \geq & c_{2}\left\|1+\left|u_{1}\right|\right\|_{L^{p}\left(\Omega, d \mu_{\varepsilon}\right)}^{p-\beta}\left\|u_{1}\right\|_{L^{p}\left(\Omega, d \mu_{\varepsilon}\right)^{N}}^{\beta}+\lambda\|u\|_{L^{p}\left(\Omega, d \mu_{\varepsilon}\right)}^{p} \\
\geq & c_{2}\left(\|1\|_{L^{p}\left(\Omega, d \mu_{\varepsilon}\right)}+\left\|u_{1}\right\|_{L^{p}\left(\Omega, d \mu_{\varepsilon}\right)^{N}}\right)^{p-\beta}\left\|u_{1}\right\|_{L^{p}\left(\Omega, d \mu_{\varepsilon}\right)^{N}}^{\beta} \\
& +\lambda\|u\|_{L^{p}\left(\Omega, d \mu_{\varepsilon}\right)}^{p} .
\end{aligned}
$$

Let $c=\min \left\{c_{2}, \lambda\right\}$ and $k=\|1\|_{L^{p}\left(\Omega, d \mu_{\varepsilon}\right)}$ then

$$
\frac{\left\langle T\left(u, u_{1}\right),\left(u, u_{1}\right)\right\rangle}{\left\|\left(u, u_{1}\right)\right\|} \geq c \frac{\left(k+\left\|u_{1}\right\|\right)^{p}}{\left\|\left(u, u_{1}\right)\right\|}\left[\frac{\left\|u_{1}\right\|}{k+\left\|u_{1}\right\|}\right]^{\beta}+c \frac{\|u\|^{p}}{\left\|\left(u, u_{1}\right)\right\|} .
$$

The right hand side converges to $\infty$ as $\left\|\left(u, u_{1}\right)\right\| \rightarrow \infty$ which gives that $T$ is coercive.

We remark that the solution is unique as a pair $\left(u, u_{1}\right) \in W_{0}^{1, p}\left(\Omega, d \mu_{\varepsilon}\right)$, i.e. there exists a unique gradient $u_{1}$ of $u$ such that $\left(u, u_{1}\right)$ is the solution. 


\subsection{Monotonicity and two-scale convergence.}

Theorem 14. Let a satisfy the conditions (48)-(50). Moreover, let $\left(v_{\varepsilon}\right)$ be a bounded sequence in $L^{p}\left(\Omega, d \mu_{\varepsilon}\right)^{N}$ which two-scale converges weakly to $v \in L^{p}(\Omega \times Y)^{N}$ and assume that $a\left(x / \varepsilon, v_{\varepsilon}(x)\right)$ two-scale converges weakly to $a_{0}(x, y) \in L^{q}(\Omega \times Y)^{N}$. Then

$$
\liminf _{\varepsilon \rightarrow 0} \int_{\Omega} a\left(x / \varepsilon, v_{\varepsilon}(x)\right) \cdot v_{\varepsilon}(x) d \mu_{\varepsilon} \geq \int_{\Omega} \int_{Y} a_{0}(x, y) \cdot v(x, y) d \mu d x
$$

and if (54) holds as equality, then $a_{0}(x, y)=a(y, v(x, y))$.

Proof. Assume the contradiction, i.e.

$$
\liminf _{\varepsilon \rightarrow 0} \int_{\Omega} a\left(x / \varepsilon, v_{\varepsilon}(x)\right) \cdot v_{\varepsilon}(x) d \mu_{\varepsilon}<\int_{\Omega} \int_{Y} a_{0}(x, y) \cdot v(x, y) d \mu d x .
$$

Then there exists a positive constant $k>0$ such that

(55) $\liminf _{\varepsilon \rightarrow 0} \int_{\Omega} a\left(x / \varepsilon, v_{\varepsilon}(x)\right) \cdot v_{\varepsilon}(x) d \mu_{\varepsilon}=\int_{\Omega} \int_{Y} a_{0}(x, y) \cdot v(x, y) d \mu d x-k$.

Let $\phi(x, y)=\psi(x) \sigma(y)$, where $\psi \in C_{0}^{\infty}(\Omega)$ and $\sigma \in C_{\text {per }}^{\infty}(Y)^{N}$. By monotonicity we have that

$$
\int_{\Omega}\left[a\left(x / \varepsilon, v_{\varepsilon}(x)\right)-a(x / \varepsilon, \phi(x, x / \varepsilon))\right] \cdot\left[v_{\varepsilon}(x)-\phi(x, x / \varepsilon)\right] d \mu_{\varepsilon} \geq 0 .
$$

This inequality consists of four terms. Using the assumption (55) on the corresponding term and passing to the limit in the other three terms gives

$$
\int_{\Omega} \int_{Y}\left[a_{0}(x, y)-a(y, \phi(x, y))\right] \cdot[v(x, y)-\phi(x, y)] d \mu d x \geq k .
$$

By density and continuity (see [5], p. 77) this inequality also holds for any $\phi \in L^{p}(\Omega \times Y)^{N}$. Let $t w(x, y)=v(x, y)-\phi(x, y), t>0$. Then after dividing by $t$ we get

$$
\int_{\Omega} \int_{Y}\left[a_{0}(x, y)-a(y, v(x, y)-t w(x, y))\right] \cdot w(x, y) d \mu d x \geq k / t .
$$

Letting $t \rightarrow 0$ gives

$$
\int_{\Omega} \int_{Y}\left[a_{0}(x, y)-a(y, v(x, y))\right] \cdot w(x, y) d \mu d x \geq \infty .
$$

Repeating the procedure for $t<0$ implies that

$$
\int_{\Omega} \int_{Y}\left[a_{0}(x, y)-a(y, v(x, y))\right] \cdot w(x, y) d \mu d x \leq-\infty .
$$

We have clearly reached a contradiction.

If (54) holds as equality we can repeat the procedure above (with $k=0$ ) and we get that

$$
\int_{\Omega} \int_{Y}\left[a_{0}(x, y)-a(y, v(x, y))\right] \cdot w(x, y) d \mu d x=0, \quad \forall w \in L^{p}(\Omega \times Y)^{N} .
$$


Hence $a_{0}(x, y)=a(y, v(x, y))$.

Theorem 15. Let $a(x, \xi)$ satisfy the conditions (48)-(50). Moreover, let $\left(v_{\varepsilon}\right)$ be a bounded sequence in $L^{p}\left(\Omega, d \mu_{\varepsilon}\right)^{N}$ such that $v_{\varepsilon}$ two-scale converges weakly to $v \in L^{p}(\Omega \times Y)^{N},\left|v_{\varepsilon}\right|^{p-2} v_{\varepsilon}$ two-scale converges weakly to $w \in L^{q}(\Omega \times Y)^{N}$ and $a\left(x / \varepsilon, v_{\varepsilon}(x)\right)$ two-scale converges weakly to $a_{0}(x, y) \in$ $L^{q}(\Omega \times Y)^{N}$. If

$$
\lim _{\varepsilon \rightarrow 0} \int_{\Omega} a\left(\frac{x}{\varepsilon}, v_{\varepsilon}(x)\right) \cdot v_{\varepsilon}(x) d \mu_{\varepsilon}=\int_{\Omega} \int_{Y} a_{0}(x, y) \cdot v(x, y) d \mu d x,
$$

then $v_{\varepsilon}$ two-scale converges strongly to $v \in L^{p}(\Omega \times Y)^{N}$.

Proof. We may rewrite the right hand side in (57) as follows:

$$
\begin{aligned}
& \lim _{\varepsilon \rightarrow 0} \int_{\Omega} a\left(x / \varepsilon, v_{\varepsilon}\right) \cdot v_{\varepsilon} d \mu_{\varepsilon} \\
& =\limsup _{\varepsilon \rightarrow 0} \int_{\Omega} k\left|v_{\varepsilon}\right|^{p} d \mu_{\varepsilon}-\limsup _{\varepsilon \rightarrow 0} \int_{\Omega} k\left|v_{\varepsilon}\right|^{p} d \mu_{\varepsilon} \\
& \quad+\lim _{\varepsilon \rightarrow 0} \int_{\Omega} a\left(\frac{x}{\varepsilon}, v_{\varepsilon}\right) \cdot v_{\varepsilon} d \mu_{\varepsilon} \\
& =\limsup _{\varepsilon \rightarrow 0} \int_{\Omega} k\left|v_{\varepsilon}\right|^{p} d \mu_{\varepsilon}+\liminf _{\varepsilon \rightarrow 0} \int_{\Omega}-k\left|v_{\varepsilon}\right|^{p} d \mu_{\varepsilon} \\
& \quad+\lim _{\varepsilon \rightarrow 0} \int_{\Omega} a\left(\frac{x}{\varepsilon}, v_{\varepsilon}\right) \cdot v_{\varepsilon} d \mu_{\varepsilon} \\
& =\limsup _{\varepsilon \rightarrow 0} \int_{\Omega} k\left|v_{\varepsilon}\right|^{p} d \mu_{\varepsilon}+\liminf _{\varepsilon \rightarrow 0} \int_{\Omega}\left[a\left(\frac{x}{\varepsilon}, v_{\varepsilon}\right)-k\left|v_{\varepsilon}\right|^{p-2} v_{\varepsilon}\right] \cdot v_{\varepsilon} d \mu_{\varepsilon} .
\end{aligned}
$$

This together with (57) gives

$$
\begin{gathered}
\limsup _{\varepsilon \rightarrow 0} \int_{\Omega} k\left|v_{\varepsilon}\right|^{p} d \mu_{\varepsilon}+\liminf _{\varepsilon \rightarrow 0} \int_{\Omega}\left[a\left(\frac{x}{\varepsilon}, v_{\varepsilon}\right)-k\left|v_{\varepsilon}\right|^{p-2} v_{\varepsilon}\right] \cdot v_{\varepsilon} d \mu_{\varepsilon} \\
\quad=\int_{\Omega} \int_{Y} k w \cdot v d \mu d x+\int_{\Omega} \int_{Y}\left(a_{0}-k w\right) \cdot v d \mu d x .
\end{gathered}
$$

For $k>0$ sufficiently small the function $a(x, \xi)-k|\xi|^{p-2} \xi$ satisfies the conditions in Theorem 14 therefore

$$
\liminf _{\varepsilon \rightarrow 0} \int_{\Omega}\left[a\left(\frac{x}{\varepsilon}, v_{\varepsilon}\right)-k\left|v_{\varepsilon}\right|^{p-2} v_{\varepsilon}\right] \cdot v_{\varepsilon} d \mu_{\varepsilon} \geq \int_{\Omega} \int_{Y}\left(a_{0}-k w\right) \cdot v d \mu d x .
$$

This and (58) implies

$$
\limsup _{\varepsilon \rightarrow 0} \int_{\Omega}\left|v_{\varepsilon}\right|^{p} d \mu_{\varepsilon} \leq \int_{\Omega} \int_{Y} w \cdot v d \mu d x .
$$

The function $|\xi|^{p-2} \xi$ satisfies the conditions in Theorem 14 which implies that

(60) $\liminf _{\varepsilon \rightarrow 0} \int_{\Omega}\left|v_{\varepsilon}\right|^{p} d \mu_{\varepsilon}=\liminf _{\varepsilon \rightarrow 0} \int_{\Omega}\left|v_{\varepsilon}\right|^{p-2} v_{\varepsilon} \cdot v_{\varepsilon} d \mu_{\varepsilon} \geq \int_{\Omega} \int_{Y} w \cdot v d \mu d x$. 
By (59) and (60) it follows that

$$
\lim _{\varepsilon \rightarrow 0} \int_{\Omega}\left|v_{\varepsilon}\right|^{p} d \mu_{\varepsilon}=\lim _{\varepsilon \rightarrow 0} \int_{\Omega}\left|v_{\varepsilon}\right|^{p-2} v_{\varepsilon} \cdot v_{\varepsilon} d \mu_{\varepsilon}=\int_{\Omega} \int_{Y} w \cdot v d \mu d x .
$$

Hence it follows by Theorem 14 that $w=|v|^{p-2} v$ and Theorem 5 gives that $v_{\varepsilon}$ two-scale converges strongly to $v \in L^{p}(\Omega \times Y)^{N}$.

\section{Homogenization of a class of monotone operators}

Let us consider the equation

(61) $-\operatorname{div}\left(a\left(x / \varepsilon, D u_{\varepsilon}\right)\right)+\lambda\left|u_{\varepsilon}\right|^{p-2} u_{\varepsilon}=f_{\varepsilon}, \quad\left(u_{\varepsilon}, D u_{\varepsilon}\right) \in W_{0}^{1, p}\left(\Omega, d \mu_{\varepsilon}\right)$,

where $a$ satisfies the conditions (48)-(50), $f_{\varepsilon} \in L^{q}\left(\Omega, d \mu_{\varepsilon}\right)$ and $\lambda>0$. We recall that by definition $\left(u_{\varepsilon}, D u_{\varepsilon}\right) \in W_{0}^{1, p}\left(\Omega, d \mu_{\varepsilon}\right)$ is a solution of (61) if the following integral identity holds:

$$
\int_{\Omega} a\left(x / \varepsilon, D u_{\varepsilon}\right) \cdot D \phi+\lambda\left|u_{\varepsilon}\right|^{p-2} u_{\varepsilon} \phi d \mu_{\varepsilon}=\int_{\Omega} f_{\varepsilon} \phi d \mu_{\varepsilon}, \forall \phi \in C_{0}^{\infty}(\Omega) .
$$

According to Theorem 13 the equation (61) has a unique solution. We will prove that the solutions $\left(u_{\varepsilon}, D u_{\varepsilon}\right)$ converge in some sense (to be specified below) to the solution $u$ of a homogenized problem associated with the Lebesgue measure:

$$
-\operatorname{div}(b(D u))+\lambda|u|^{p-2} u=f, \quad u \in W_{0}^{1, p}(\Omega)
$$

where $f \in L^{q}(\Omega)$ and the homogenized operator $b$ is defined as

$$
b(\xi) \stackrel{\text { def }}{=} \int_{Y} a\left(y, \xi+v_{\xi}(y)\right) d \mu
$$

and $v_{\xi} \in V_{\text {pot }}^{p}$ is the solution of the periodic problem:

$$
\int_{Y} a\left(y, \xi+v_{\xi}(y)\right) \cdot D w(y) d \mu=0, \quad \forall w \in C_{\mathrm{per}}^{\infty}(Y) .
$$

By definition $u \in W_{0}^{1, p}(\Omega)$ is a solution of (63) if the following integral identity holds:

$$
\int_{\Omega} b(D u) \cdot D \phi+\lambda|u|^{p-2} u \phi d x=\int_{\Omega} f \phi d x, \quad \forall \phi \in C_{0}^{\infty}(\Omega) .
$$

Theorem 16. Assume that the measure $\mu$ is non-degenerate and that $\left(f_{\varepsilon}\right)$ converges strongly to $f \in L^{q}(\Omega, d x)$. Let $\left(u_{\varepsilon}, D u_{\varepsilon}\right)$ be the sequence of solutions of $(61)$. Then $\left(u_{\varepsilon}\right)$ converges strongly to $u$, where $u$ is to the solution of the homogenized equation (63) and $\left(D u_{\varepsilon}\right)$ two-scale converges strongly to $D u+u_{1}(x, y)$ where $u_{1} \in L^{p}\left(\Omega, V_{\text {pot }}^{p}\right)$ is the solution of the periodic problem:

$$
\int_{Y} a\left(y, D u(x)+u_{1}(x, y)\right) \cdot D w(y) d \mu=0, \quad \forall w \in C_{p e r}^{\infty}(Y) .
$$


Proof. By density it is possible to chose $\left(u_{\varepsilon}, D u_{\varepsilon}\right) \in W_{0}^{1, p}\left(\Omega, d \mu_{\varepsilon}\right)$ as a test function in (62) which gives

$$
\int_{\Omega} a\left(\frac{x}{\varepsilon}, D u_{\varepsilon}\right) \cdot D u_{\varepsilon}+\lambda\left|u_{\varepsilon}\right|^{p} d \mu_{\varepsilon}=\int_{\Omega} f_{\varepsilon} u_{\varepsilon} d \mu_{\varepsilon} .
$$

By using the monotonicity assumption (50) on $a$ and the Hölder inequality we obtain that

$$
\begin{aligned}
\lambda \int_{\Omega}\left|u_{\varepsilon}\right|^{p} d \mu_{\varepsilon} & \leq \int_{\Omega} c_{2}\left(1+\left|D u_{\varepsilon}\right|\right)^{p-\beta}\left|D u_{\varepsilon}\right|^{\beta}+\lambda\left|u_{\varepsilon}\right|^{p} d \mu_{\varepsilon} \\
& \leq \int_{\Omega} f_{\varepsilon} u_{\varepsilon} d \mu_{\varepsilon} \leq\left\|f_{\varepsilon}\right\|_{L^{q}\left(\Omega, d \mu_{\varepsilon}\right)}\left\|u_{\varepsilon}\right\|_{L^{p}\left(\Omega, d \mu_{\varepsilon}\right)} .
\end{aligned}
$$

Since $\left(f_{\varepsilon}\right)$ is strongly convergent in $L^{q}\left(\Omega, d \mu_{\varepsilon}\right)$ and thus bounded in $L^{q}\left(\Omega, d \mu_{\varepsilon}\right)$ we see that sequence $\left(u_{\varepsilon}\right)$ is bounded in $L^{p}\left(\Omega, d \mu_{\varepsilon}\right)$. Let us now prove that $\left(D u_{\varepsilon}\right)$ is bounded in $L^{p}\left(\Omega, d \mu_{\varepsilon}\right)^{N}$. Indeed, the fact that $\left(u_{\varepsilon}\right)$ is bounded in $L^{p}\left(\Omega, d \mu_{\varepsilon}\right)$ together with (65) implies that there exists a constant $K$ such that

$$
\int_{\Omega}\left(1+\left|D u_{\varepsilon}\right|\right)^{p-\beta}\left|D u_{\varepsilon}\right|^{\beta} d \mu_{\varepsilon} \leq K .
$$

The reversed Hölder inequality (with the dual exponents $p /(p-\beta)$ and $0<p / \beta<1$ ) gives that

$$
\begin{gathered}
\left(\|1\|_{L^{p}\left(\Omega, d \mu_{\varepsilon}\right)}+\left\|D u_{\varepsilon}\right\|_{L^{p}\left(\Omega, d \mu_{\varepsilon}\right)^{N}}\right)^{p-\beta}\left\|D u_{\varepsilon}\right\|_{L^{p}\left(\Omega, d \mu_{\varepsilon}\right)^{N}}^{\beta} \\
\leq\left\|1+\left|D u_{\varepsilon}\right|\right\|_{L^{p}\left(\Omega, d \mu_{\varepsilon}\right)}^{p-\beta}\left\|D u_{\varepsilon}\right\|_{L^{p}\left(\Omega, d \mu_{\varepsilon}\right)^{N}}^{\beta} \leq K .
\end{gathered}
$$

Let $c=\|1\|_{L^{p}\left(\Omega, d \mu_{\varepsilon}\right)}$ then

$$
\left(c+\left\|D u_{\varepsilon}\right\|_{L^{p}\left(\Omega, d \mu_{\varepsilon}\right)^{N}}\right)^{p-\beta}\left\|D u_{\varepsilon}\right\|_{L^{p}\left(\Omega, d \mu_{\varepsilon}\right)^{N}}^{\beta} \leq K .
$$

If $\left\|D u_{\varepsilon}\right\|_{L^{p}\left(\Omega, d \mu_{\varepsilon}\right)^{N}} \leq c$ we are done so let us assume that $\left\|D u_{\varepsilon}\right\|_{L^{p}\left(\Omega, d \mu_{\varepsilon}\right)^{N}} \geq$ c. Then

$$
\begin{aligned}
& \left(2\left\|D u_{\varepsilon}\right\|_{L^{p}\left(\Omega, d \mu_{\varepsilon}\right)^{N}}\right)^{p-\beta}\left\|D u_{\varepsilon}\right\|_{L^{p}\left(\Omega, d \mu_{\varepsilon}\right)^{N}}^{\beta} \\
& \quad \leq\left(c+\left\|D u_{\varepsilon}\right\|_{L^{p}\left(\Omega, d \mu_{\varepsilon}\right)^{N}}\right)^{p-\beta}\left\|D u_{\varepsilon}\right\|_{L^{p}\left(\Omega, d \mu_{\varepsilon}\right)^{N}}^{\beta} \leq K .
\end{aligned}
$$

Hence $\left\|D u_{\varepsilon}\right\|_{L^{p}\left(\Omega, d \mu_{\varepsilon}\right)^{N}}^{p} \leq K 2^{\beta-p}$. It is also clear that the sequence $\left(\left|u_{\varepsilon}\right|^{p-2} u_{\varepsilon}\right)$ is bounded in $L^{q}\left(\Omega, d \mu_{\varepsilon}\right)$. Moreover the continuity assumption (49) implies that

$$
\left|a\left(x / \varepsilon, D u_{\varepsilon}\right)\right| \leq c_{1}\left(1+\left|D u_{\varepsilon}\right|\right)^{p-1-\alpha}\left|D u_{\varepsilon}\right|^{\alpha} \leq c_{1}\left(1+\left|D u_{\varepsilon}\right|\right)^{p-1}
$$

which gives that $a\left(x / \varepsilon, D u_{\varepsilon}\right)$ is bounded in $L^{q}\left(\Omega, d \mu_{\varepsilon}\right)^{N}$ since $\left(D u_{\varepsilon}\right)$ is bounded in $L^{p}\left(\Omega, d \mu_{\varepsilon}\right)^{N}$.

By Theorem 3 there exist a subsequence (still denoted by $\varepsilon$ ) such that: 
1. There exists a $u \in L^{p}(\Omega \times Y)$ such that $u_{\varepsilon}$ two-scale converges weakly to $u$.

2. There exists a $z \in L^{p}(\Omega \times Y)^{N}$ such that $D u_{\varepsilon}$ two-scale converges weakly to $z$.

3. There exists a $w \in L^{q}(\Omega \times Y)$ such that $\left|u_{\varepsilon}\right|^{p-2} u_{\varepsilon}$ two-scale converges weakly to $w$.

4. There exists a $a_{0} \in L^{q}(\Omega \times Y)^{N}$ such that $a\left(x / \varepsilon, D u_{\varepsilon}\right)$ two-scale converges weakly to $a_{0}$.

By Theorem 12 it follows that $u(x, y)=u(x) \in W_{0}^{1, p}(\Omega)$ (thus $u_{\varepsilon} \rightarrow$ $u$ weakly in $\left.L^{p}\left(\Omega, d \mu_{\varepsilon}\right)\right)$ and $z(x, y)=D u(x)+u_{1}(x, y)$, where $u_{1} \in$ $L^{p}\left(\Omega, V_{\mathrm{pot}}^{p}\right)$.

If we pass to the limit in (62) we obtain that

$$
\begin{gathered}
\int_{\Omega} \int_{Y} a_{0}(x, y) \cdot D \phi(x) d \mu d x+\lambda \int_{\Omega} \int_{Y} w(x, y) \phi(x) d \mu d x \\
=\int_{\Omega} f(x) \phi(x) d x, \quad \forall \phi \in C_{0}^{\infty}(\Omega) .
\end{gathered}
$$

Moreover, if $\phi_{1} \in C_{0}^{\infty}(\Omega)$ and $w_{1} \in C_{\text {per }}^{\infty}(Y)$, then

$$
\begin{aligned}
\varepsilon \int_{\Omega} f_{\varepsilon} & \phi_{1}(x) w_{1}\left(\frac{x}{\varepsilon}\right) d \mu_{\varepsilon}-\varepsilon \lambda \int_{\Omega}\left|u_{\varepsilon}\right|^{p-2} u_{\varepsilon} \phi_{1}(x) w_{1}(x / \varepsilon) d \mu_{\varepsilon} \\
= & \varepsilon \int_{\Omega} a\left(\frac{x}{\varepsilon}, D u_{\varepsilon}\right) \cdot D\left(\phi_{1}(x) w_{1}(x / \varepsilon)\right) d \mu_{\varepsilon} \\
= & \varepsilon \int_{\Omega} a\left(\frac{x}{\varepsilon}, D u_{\varepsilon}\right) \cdot w_{1}(x / \varepsilon) D \phi_{1}(x) d \mu_{\varepsilon} \\
& +\int_{\Omega} a\left(\frac{x}{\varepsilon}, D u_{\varepsilon}\right) \cdot \phi_{1}(x) D w_{1}(x / \varepsilon) d \mu_{\varepsilon} .
\end{aligned}
$$

By passing to the limit we obtain

$$
\int_{\Omega} \int_{Y} a_{0}(x, y) \cdot \phi_{1}(x) D w_{1}(y) d \mu d x=0, \forall \phi_{1} \in C_{0}^{\infty}(\Omega), \forall w_{1} \in C_{\mathrm{per}}^{\infty}(Y) .
$$

Taking (66) and (67) into account together with a density argument gives that $a_{0}$ and $w$ satisfies the following equality:

$$
\int_{\Omega} \int_{Y} a_{0}(x, y) \cdot\left(D v(x)+v_{1}(x, y)\right) d \mu d x+\lambda \int_{\Omega} \int_{Y} w v d \mu d x=\int_{\Omega} f v d x,
$$

for any $v \in W_{0}^{1, p}(\Omega)$ and any $v_{1} \in L^{p}\left(\Omega, V_{\text {pot }}^{p}\right)$. Choosing $v=u$ and $v_{1}=u_{1}$ in this equality gives

$$
\int_{\Omega} \int_{Y} a_{0}(x, y) \cdot\left(D u(x)+u_{1}(x, y)\right) d \mu d x+\lambda \int_{\Omega} \int_{Y} w u d \mu d x=\int_{\Omega} f u d x .
$$


By (64) we get

(70)

$\lim _{\varepsilon \rightarrow 0} \int_{\Omega} a\left(\frac{x}{\varepsilon}, D u_{\varepsilon}\right) \cdot D u_{\varepsilon}+\lambda\left|u_{\varepsilon}\right|^{p-2} u_{\varepsilon} u_{\varepsilon} d \mu_{\varepsilon}=\lim _{\varepsilon \rightarrow 0} \int_{\Omega} f_{\varepsilon} u_{\varepsilon} d \mu_{\varepsilon}=\int_{\Omega} f u d x$.

It now follows from (69) and (70) that

(71) $\lim _{\varepsilon \rightarrow 0}\left(\int_{\Omega} a\left(x / \varepsilon, D u_{\varepsilon}\right) \cdot D u_{\varepsilon} d \mu_{\varepsilon}+\lambda \int_{\Omega}\left|u_{\varepsilon}\right|^{p-2} u_{\varepsilon} u_{\varepsilon} d \mu_{\varepsilon}\right)$

$=\int_{\Omega} \int_{Y} a_{0}(x, y) \cdot\left(D u(x)+u_{1}(x, y)\right) d \mu d x+\lambda \int_{\Omega} \int_{Y} w u d \mu d x$.

Theorem 14 implies that

(72) $\liminf _{\varepsilon \rightarrow 0} \int_{\Omega} a\left(x / \varepsilon, D u_{\varepsilon}\right) \cdot D u_{\varepsilon} d \mu_{\varepsilon} \geq \int_{\Omega} \int_{Y} a_{0}(x, y)$ $\times\left(D u(x)+u_{1}(x, y)\right) d \mu d x$,

$$
\liminf _{\varepsilon \rightarrow 0} \int_{\Omega}\left|u_{\varepsilon}\right|^{p-2} u_{\varepsilon} u_{\varepsilon} d \mu_{\varepsilon} \geq \int_{\Omega} \int_{Y} w u d \mu d x .
$$

From (71), (72) and (73) we see that

$$
\begin{aligned}
\lim _{\varepsilon \rightarrow 0} \int_{\Omega} a\left(x / \varepsilon, D u_{\varepsilon}\right) \cdot D u_{\varepsilon} d \mu_{\varepsilon} & =\int_{\Omega} \int_{Y} a_{0}(x, y) \cdot\left(D u(x)+u_{1}(x, y)\right) d \mu d x, \\
\lim _{\varepsilon \rightarrow 0} \int_{\Omega}\left|u_{\varepsilon}\right|^{p-2} u_{\varepsilon} u_{\varepsilon} d \mu_{\varepsilon} & =\int_{\Omega} \int_{Y} w u d \mu d x .
\end{aligned}
$$

Theorem 14 now gives that $a_{0}(x, y)=a\left(y, D u(x)+u_{1}(x, y)\right)$ and $w(x, y)=$ $|u|^{p-2} u$ and by Theorem 15 it follows that $\left(u_{\varepsilon}\right)$ converges strongly to $u$ and $\left(D u_{\varepsilon}\right)$ two-scale converges strongly to $D u+u_{1}(x, y)$. Moreover, we have that $u$ and $u_{1}$ satisfy the following equality, see (68):

$$
\begin{aligned}
& \int_{\Omega} \int_{Y} a\left(y, D u(x)+u_{1}(x, y)\right) \cdot\left(D v(x)+v_{1}(x, y)\right) d \mu d x \\
& \quad+\lambda \int_{\Omega} \int_{Y}|u|^{p-2} u v d \mu d x=\int_{\Omega} f v d x
\end{aligned}
$$

for any $v \in W_{0}^{1, p}(\Omega)$ and any $v_{1} \in L^{p}\left(\Omega, V_{\text {pot }}^{p}\right)$.

By separation of variables this system is equivalent to: First find $u \in$ $W_{0}^{1, p}(\Omega)$ such that

$$
-\operatorname{div}(b(D u))+\lambda|u|^{p-2} u=f,
$$

where $b$ is defined as

$$
b(\xi) \stackrel{\text { def }}{=} \int_{Y} a\left(y, \xi+v_{\xi}(y)\right) d \mu
$$

and $v_{\xi} \in V_{\text {pot }}^{p}$ is the solution of the periodic problem:

$$
\int_{Y} a\left(y, \xi+v_{\xi}(y)\right) \cdot D w(y) d \mu=0, \quad \forall w \in C_{\mathrm{per}}^{\infty}(Y) .
$$


Secondly $u_{1}(x, y)=v_{D u(x)}(y)$. We so far only proved the theorem up to a subsequence. However, the properties of the homogenized operator $b$ (see the next section) implies that the solution of the homogenized equation (63) is unique and hence the theorem hold for the whole sequence.

\section{Some properties of the homogenized operator}

The homogenized operator $b$ was defined as

$$
b(\xi) \stackrel{\text { def }}{=} \int_{Y} a\left(y, \xi+v_{\xi}(y)\right) d \mu
$$

where $v_{\xi} \in V_{\text {pot }}^{p}$ is the solution of the periodic problem:

$$
\int_{Y} a\left(y, \xi+v_{\xi}(y)\right) \cdot D w(y) d \mu=0, \quad \forall w \in C_{\mathrm{per}}^{\infty}(Y) .
$$

Theorem 17. The homogenized operator $b$ defined as in (74) has the following properties:

(i) $b$ is strictly monotone, more precisely there exists a constant $\widetilde{c}_{2}>0$ such that

$$
\left(b\left(\xi_{1}\right)-b\left(\xi_{2}\right)\right) \cdot\left(\xi_{1}-\xi_{2}\right) \geq \widetilde{c}_{2}\left(1+\left|\xi_{1}\right|+\left|\xi_{2}\right|\right)^{p-\beta}\left|\xi_{1}-\xi_{2}\right|^{\beta},
$$

for any $\xi_{1}, \xi_{2} \in \mathbb{R}^{N}$.

(ii) $b$ is continuous, more precisely there exists a constant $\widetilde{c}_{1}>0$ such that

$$
\left|b\left(\xi_{1}\right)-b\left(\xi_{2}\right)\right| \leq \widetilde{c_{1}}\left(1+\left|\xi_{1}\right|+\left|\xi_{2}\right|\right)^{p-1-\alpha /(\beta-\alpha)}\left|\xi_{1}-\xi_{2}\right|^{\alpha /(\beta-\alpha)},
$$

for any $\xi_{1}, \xi_{2} \in \mathbb{R}^{N}$.

(iii) $b(0)=0$.

Proof. (i) We have

$$
\begin{aligned}
\left(b\left(\xi_{1}\right)-b\left(\xi_{2}\right)\right) \cdot\left(\xi_{1}-\xi_{2}\right)= & \int_{Y}\left[a\left(y, \xi_{1}+v_{\xi_{1}}(y)\right)-a\left(y, \xi_{2}+v_{\xi_{2}}(y)\right)\right] \\
& \times\left[\xi_{1}+v_{\xi_{1}}(y)-\left(\xi_{2}+v_{\xi_{2}}(y)\right)\right] d \mu
\end{aligned}
$$

By the monotonicity condition (50) we obtain

$$
\begin{aligned}
\left(b\left(\xi_{1}\right)-b\left(\xi_{2}\right)\right) \cdot\left(\xi_{1}-\xi_{2}\right) \geq & c_{2} \int_{Y}\left(1+\left|\xi_{1}+v_{\xi_{1}}\right|+\left|\xi_{2}+v_{\xi_{2}}\right|\right)^{p-\beta} \\
& \times\left|\xi_{1}+v_{\xi_{1}}-\left(\xi_{2}+v_{\xi_{2}}\right)\right|^{\beta} d \mu
\end{aligned}
$$

The reversed Hölder inequality (with the dual exponents $0<p / \beta<1$ and $p /(p-\beta))$ implies that

$$
\begin{aligned}
& \left(b\left(\xi_{1}\right)-b\left(\xi_{2}\right)\right) \cdot\left(\xi_{1}-\xi_{2}\right) \\
& \quad \geq c_{2}\left(\int_{Y}\left(1+\left|\xi_{1}+v_{\xi_{1}}\right|+\left|\xi_{2}+v_{\xi_{2}}\right|\right)^{p} d \mu\right)^{(p-\beta) / p}
\end{aligned}
$$




$$
\times\left(\int_{Y}\left|\xi_{1}-\xi_{2}+v_{\xi_{1}}-v_{\xi_{2}}\right|^{p} d \mu\right)^{\beta / p} .
$$

Let us now consider the second factor in the right hand side of this inequality. Letting $\eta=\left|\xi_{1}-\xi_{2}\right|^{-1}\left(\xi_{1}-\xi_{2}\right)$ and $v=\left|\xi_{1}-\xi_{2}\right|^{-1}\left(v_{\xi_{1}}-v_{\xi_{2}}\right)$ gives

$$
\text { (78) } \begin{aligned}
\left.\int_{Y} \mid \xi_{1}-\xi_{2}+v_{\xi_{1}}-v_{\xi_{2}}\right)\left.\right|^{p} d \mu & =\left|\xi_{1}-\xi_{2}\right|^{p} \int_{Y}|\eta+v|^{p} d \mu \\
& \geq\left|\xi_{1}-\xi_{2}\right|^{p} \min _{w \in V_{\text {pot }}^{p}} \int_{Y}|\eta+w|^{p} d \mu .
\end{aligned}
$$

Let $S_{N}$ be the unit sphere in $\mathbb{R}^{N}$ and define $m: S_{N} \rightarrow \mathbb{R}$ as

$$
m(\eta)=\min _{w \in V_{\mathrm{pot}}^{p}} \int_{Y}|\eta+w(y)|^{p} d \mu .
$$

Since the measure $\mu$ is non-degenerate we have that $m(\eta)>0$. The continuous function $m$ attains its minimum as $\eta$ ranges over the unit sphere in $\mathbb{R}^{N}$. This implies that there exists a constant $k>0$ such that $m(\eta) \geq k$ for every $\eta$. This together with (77) and (78) implies that there exists $c>0$ such that

$$
\begin{aligned}
& \left(b\left(\xi_{1}\right)-b\left(\xi_{2}\right)\right) \cdot\left(\xi_{1}-\xi_{2}\right) \\
& \quad \geq c\left(\int_{Y}\left(1+\left|\xi_{1}+v_{\xi_{1}}\right|+\left|\xi_{2}+v_{\xi_{2}}\right|\right)^{p} d \mu\right)^{(p-\beta) / p}\left|\xi_{1}-\xi_{2}\right|^{\beta} .
\end{aligned}
$$

The Minkowski inequality gives

$$
\geq c\left(1+\left(\int_{Y}\left|\xi_{1}+v_{\xi_{1}}\right|^{p} d \mu\right)^{1 / p}+\left(\int_{Y}\left|\xi_{2}+v_{\xi_{2}}\right|^{p} d \mu\right)^{1 / p}\right)^{p-\beta}\left|\xi_{1}-\xi_{2}\right|^{\beta} .
$$

If we prove that there exists a constant $\widetilde{k}>0$ such that

$$
\int_{Y}\left|\xi+v_{\xi}\right|^{p} d \mu \leq \widetilde{k}\left(1+|\xi|^{p}\right)
$$

Then it follows from (79) that there exists a constant $\widetilde{c}_{2}>0$ such that

$$
\left(b\left(\xi_{1}\right)-b\left(\xi_{2}\right)\right) \cdot\left(\xi_{1}-\xi_{2}\right) \geq \widetilde{c}_{2}\left(1+\left|\xi_{1}\right|+\left|\xi_{2}\right|\right)^{p-\beta}\left|\xi_{1}-\xi_{2}\right|^{\beta}
$$

and the proof is complete. Thus it remains to prove (80). By (51) we get

$$
\begin{aligned}
\int_{Y}\left|\xi+v_{\xi}\right|^{p} d \mu & \leq c_{4} \int_{Y} 1+a\left(x, \xi+v_{\xi}\right) \cdot\left(\xi+v_{\xi}\right) d \mu \\
& =c_{4} \int_{Y} 1+a\left(x, \xi+v_{\xi}\right) \cdot \xi d \mu \\
& \leq c_{4} \int_{Y} 1+\left|a\left(x, \xi+v_{\xi}\right)\right||\xi| d \mu
\end{aligned}
$$




$$
\begin{aligned}
& \leq c_{4} \int_{Y} 1+c_{3}\left(1+\left|\xi+v_{\xi}\right|^{p-1}\right)|\xi| d \mu \\
& =c_{4}+c_{4} c_{3} \int_{Y}\left(1+\left|\xi+v_{\xi}\right|^{p-1}\right)|\xi| d \mu .
\end{aligned}
$$

The Young inequality for numbers $\left(a b \leq 1 / s \eta^{s} a^{s}+1 / t \eta^{-t} b^{t}\right.$, where $1 / s+1 / t=1)$ applied to the integrand in the right hand side implies

$$
\begin{aligned}
\left(1+\left|\xi+v_{\xi}\right|^{p-1}\right)|\xi| & \leq \frac{1}{q} \eta^{q}\left(1+\left|\xi+v_{\xi}\right|^{p-1}\right)^{q}+\frac{1}{p} \eta^{-p}|\xi|^{p} \\
& \leq \frac{1}{q} \eta^{q} 2^{q-1}\left(1+\left|\xi+v_{\xi}\right|^{p}\right)+\frac{1}{p} \eta^{-p}|\xi|^{p}
\end{aligned}
$$

This together with (81) gives that there exists a constant $c>0$ for which

$$
\begin{aligned}
\int_{Y}\left|\xi+v_{\xi}\right|^{p} d \mu & \leq c_{4}+c_{4} c_{3} \int_{Y} \frac{1}{q} \eta^{q} 2^{q-1}\left(1+\left|\xi+v_{\xi}\right|^{p}\right)+\frac{1}{p} \eta^{-p}|\xi|^{p} d \mu \\
& =c+c \eta^{q}+c \eta^{q} \int_{Y}\left|\xi+v_{\xi}\right|^{p} d \mu+c \eta^{-p}|\xi|^{p} .
\end{aligned}
$$

Finally, by choosing $\eta$ sufficiently small we obtain that there exists a constant $\widetilde{k}>0$ such that

$$
\int_{Y}\left|\xi+v_{\xi}\right|^{p} d \mu \leq \widetilde{k}\left(1+|\xi|^{p}\right) .
$$

(ii) By Jensen's inequality, the continuity assumption (49) and the Hölder inequality we have that

$$
\begin{aligned}
\left|b\left(\xi_{1}\right)-b\left(\xi_{2}\right)\right|^{q}= & \left|\int_{Y} a\left(y, \xi_{1}+v_{\xi_{1}}(y)\right)-a\left(y, \xi_{2}+v_{\xi_{2}}(y)\right) d \mu\right|^{q} \\
\leq & \int_{Y}\left|a\left(y, \xi_{1}+v_{\xi_{1}}(y)\right)-a\left(y, \xi_{2}+v_{\xi_{2}}(y)\right)\right|^{q} d \mu \\
\leq & c_{1}^{q} \int_{Y}\left(1+\left|\xi_{1}+v_{\xi_{1}}(y)\right|+\left|\xi_{2}+v_{\xi_{2}}(y)\right|\right)^{(p-1-\alpha) q} \\
& \times\left|\xi_{1}+v_{\xi_{1}}(y)-\xi_{2}-v_{\xi_{2}}(y)\right|^{\alpha q} d \mu \\
\leq & c_{1}^{q}\left(\int_{Y}\left(1+\left|\xi_{1}+v_{\xi_{1}}(y)\right|+\left|\xi_{2}+v_{\xi_{2}}(y)\right|\right)^{p} d \mu\right)^{(p-\alpha q) / p} \\
& \times\left(\int_{Y}\left|\xi_{1}+v_{\xi_{1}}(y)-\xi_{2}-v_{\xi_{2}}(y)\right|^{p} d \mu\right)^{\alpha q / p} .
\end{aligned}
$$

By using (80) there exists a $c>0$ such that

(82) $\left|b\left(\xi_{1}\right)-b\left(\xi_{2}\right)\right|^{q} \leq c\left(1+\left|\xi_{1}\right|^{p}+\left|\xi_{2}\right|^{p}\right)^{(p-\alpha q) / p}$

$$
\times\left(\int_{Y}\left|\xi_{1}+v_{\xi_{1}}(y)-\xi_{2}-v_{\xi_{2}}(y)\right|^{p} d \mu\right)^{\alpha q / p} .
$$


Now we want to estimate the integral in this inequality. This is done by using the same arguments as in [3] on (page 32 in part A) which gives that

$$
\begin{aligned}
& \int_{Y}\left|\xi_{1}+v_{\xi_{1}}(y)-\xi_{2}-v_{\xi_{2}}(y)\right|^{p} d \mu \\
& \quad \leq c\left(1+\left|\xi_{1}\right|^{p}+\left|\xi_{2}\right|^{p}\right)^{(\beta-\alpha-1) /(\beta-\alpha)}\left|\xi_{1}-\xi_{2}\right|^{p /(\beta-\alpha)},
\end{aligned}
$$

for every $\xi_{1}, \xi_{2} \in \mathbb{R}^{N}$ and where $c$ is some strictly positive constant. This together with (82) implies that there exists a $c>0$ such that

$$
\begin{aligned}
\left|b\left(\xi_{1}\right)-b\left(\xi_{2}\right)\right|^{q} \leq & c\left(1+\left|\xi_{1}\right|^{p}+\left|\xi_{2}\right|^{p}\right)^{(p-\alpha q) / p} \\
& \times\left(1+\left|\xi_{1}\right|^{p}+\left|\xi_{2}\right|^{p}\right)^{\alpha q(\beta-\alpha-1) /[p(\beta-\alpha)]}\left|\xi_{1}-\xi_{2}\right|^{\alpha q /(\beta-\alpha)} \\
= & c\left(1+\left|\xi_{1}\right|^{p}+\left|\xi_{2}\right|^{p}\right)^{(p-1-\alpha /(\beta-\alpha)) q / p}\left|\xi_{1}-\xi_{2}\right|^{\alpha q /(\beta-\alpha)} .
\end{aligned}
$$

From this it is clear that there exists a constant $\widetilde{c_{1}}>0$ such that

$$
\left|b\left(\xi_{1}\right)-b\left(\xi_{2}\right)\right| \leq \widetilde{c_{1}}\left(1+\left|\xi_{1}\right|+\left|\xi_{2}\right|\right)^{p-1-\alpha /(\beta-\alpha)}\left|\xi_{1}-\xi_{2}\right|^{\alpha /(\beta-\alpha)} .
$$

(iii) $b(0)=0$ follows directly from that $a(x, 0)=0$.

A Final Remark. After completing this paper it has come to our knowledge that Professor V.V. Zhikov and his team have recently done some work devoted to homogenization of monotone operators. We have not succeeded to obtain knowledge of the content of this still not printed work. The only information we have been able to get is that their results will be printed in Russian (and later translated into English) in the proceeding of the Petrovskii seminar, N24.

Acknowledgments. We thank the careful referee and Professor L.-E. Persson for several suggestions, which have improved the final version of this paper. Moreover, we want to thank NorFA for their mobility support.

\section{References}

[1] G. Allaire, Homogenization and two-scale convergence, SIAM J. Math. Anal., 23 (1992), 1482-1518.

[2] G. Bouchitté and I. Fragalà, Homogenization of thin structures by twoscale method with respect to measures, SIAM J. Math. Anal., 32 (6) (2001), 1198-1226.

[3] J. Byström, Some mathematical and engineering aspects of the homogenization theory, Ph.D. thesis, Dept. of Math., Luleå University of Technology, Luleå, Sweden, 2002.

[4] G. Dal Maso, An Introduction to $\Gamma$-Convergence, Birkhäuser, Boston, 1993. 
[5] I. Ekeland and R. Temam, Convex Analysis and Variational Problems, North-Holland, Amsterdam, 1976.

[6] G. Nguetseng, D. Lukkassen and P. Wall, Two-scale convergence, International J. of Pure and Appl Math., 2 (1) (2002), 35-86.

[7] G. Nguetseng, A general convergence result for a functional related to the theory of homogenization, SIAM J. Math. Anal, 20 (3) (1989), 608-623.

[8] W. Rudin, Functional Analysis, 2nd edition, McGraw-Hill, New York, 1991.

[9] J. Van Tiel, Convex Analysis: An Introductory Text, Wiley, New York, 1984.

[10] V. V. Zhikov, Connectedness and homogenization - Examples of fractal conductivity, Sb. Math., 187 (8) (1996), 3-40.

[11] V. V. Zhikov, Weighted Sobolev spaces, Sb. Math., 189 (8) (1997), 1139-1170.

[12] V. V. Zhikov, On a homogenization technique for variational problems, Funct. Anal. Appl., 33 (1) (1999), 11-24.

[13] V. V. Zhikov, On an extension and an application of the two-scale convergence method, Sb. Math., 191 (7-8) (2000), 973-1014.

[14] V. V. Zhikov, Homogenization of elasticity problems on singular structures, Izv. Math., 66 (2) (2002), 299-365.

[15] V. V. Zhikov, On two-scale convergence, Proceeding of Petrovsky Seminar, 23 (2003).

Narvik University College

N-8505 Narvik

Norway

(E-mail : dl@hin.no)

Department of Mathematics

Luleå University of Technology

S-971 87 Luleå

Sweden

(E-mail : wall@sm.luth.se) 


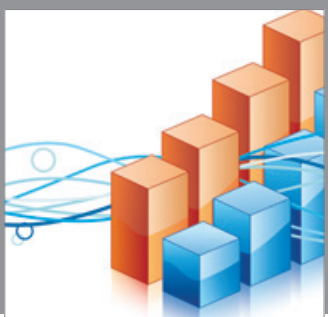

Advances in

Operations Research

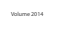

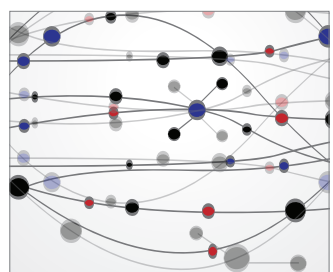

\section{The Scientific} World Journal
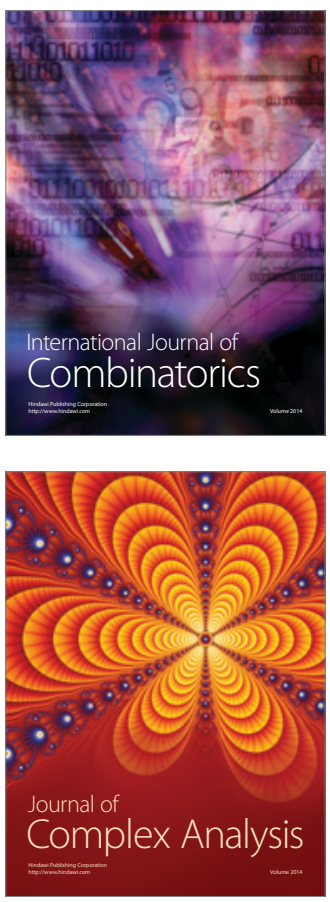

International Journal of

Mathematics and

Mathematical

Sciences
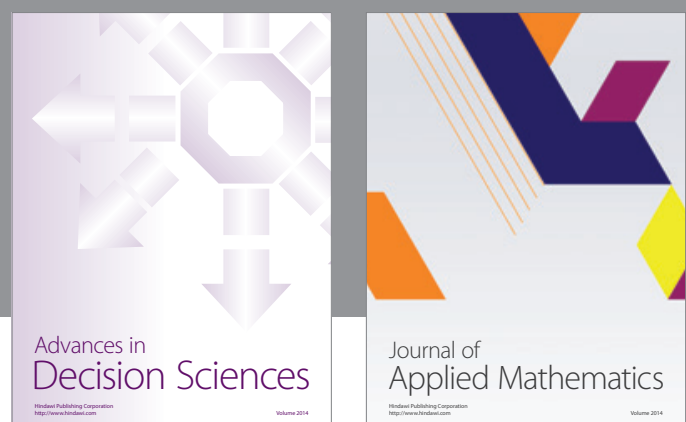

Journal of

Applied Mathematics
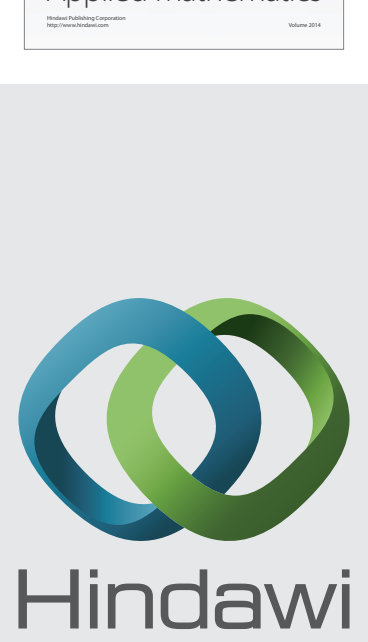

Submit your manuscripts at http://www.hindawi.com
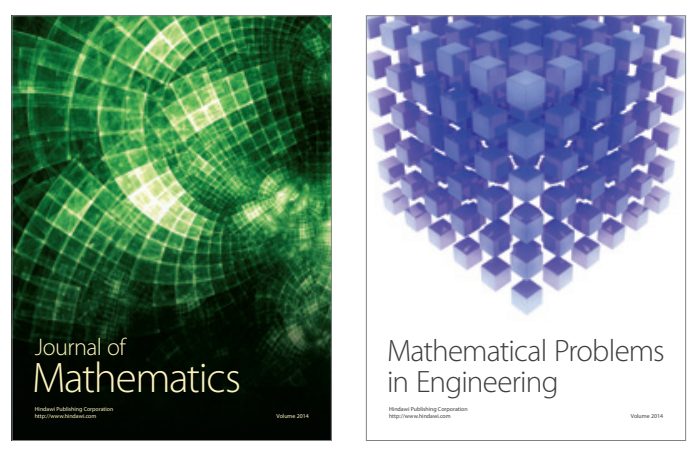

Mathematical Problems in Engineering
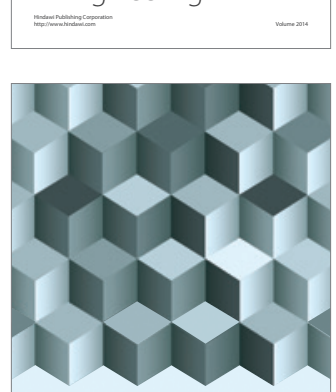

Journal of

Function Spaces
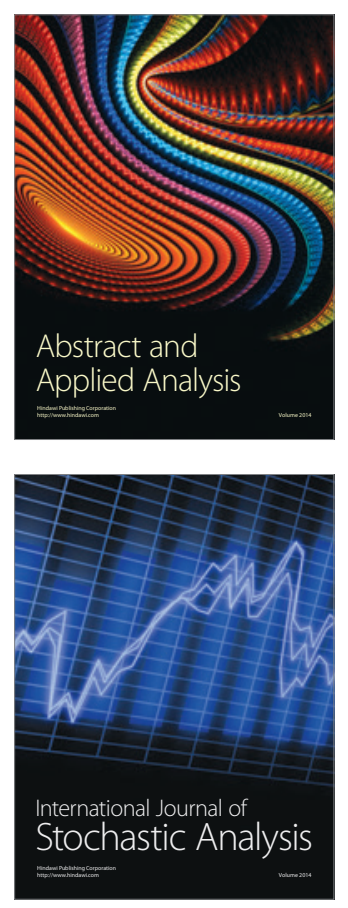

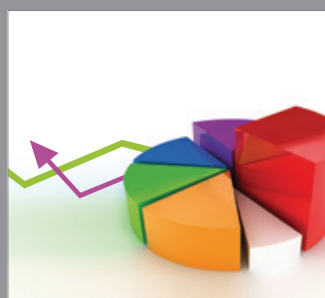

ournal of

Probability and Statistics

Promensencen
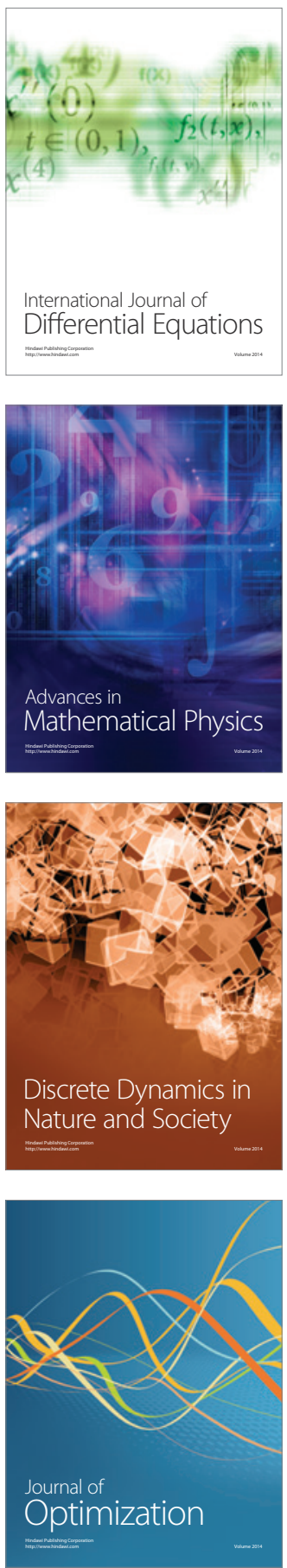